\title{
GENERALIZED EDGE CONNECTIVITY IN GRAPHS
}

\author{
By \\ Kamal P. Hennayake \\ DISSERTATION \\ Submitted to \\ The Eberly College of Arts and Sciences \\ at \\ West Virginia University \\ in partial fulfillment of the requirements \\ for the degree of \\ Doctor of Philosophy \\ in \\ Mathematics \\ Department of Mathematics \\ Morgantown, West Virginia \\ 1998
}




\begin{abstract}
The edge-connectivity $\lambda$ of a connected graph is the minimum number of edges whose deletion produces a graph with two components. For an integer $l>1$, the $l$-edgeconnectivity of a graph with $|V| \geq l$, denoted by $\lambda_{l}$, is the smallest number of edges whose removal results in a graph with $l$ components. The strength of $G$, denoted by $\bar{\lambda}(G)$, is the maximum value of $\lambda(H)$, where $H$ runs over all subgraphs of $G$. In this dissertation we study lower bounds of $\lambda_{l}$ and optimal graphs that reach the lower bound. Former results in [SIAM J. Appl. Math., 34 (1978) 657-665] are extended. We also present an optimal model of interconnection network $G$ with a given $\lambda_{l}(G)$ such that $\lambda(G)$ is maximized while $|E(G)|$ is minimized. Further we investigate the relationship between $\lambda_{l}$ and $\bar{\lambda}$, especially the extremal cases. A structural characterization of the extremal when $\lambda_{2}=2$ and arbitrary $l \geq 2$ is found.
\end{abstract}

For an integer $k>1$, a graph is $(k, l)$-edge-connected if the $l$-edge-connectivity of $G$ is at least $k$. A graph $G$ is minimally $(k, l)$-edge connected if $\lambda_{l}(G) \geq k$ but for any edge $e \in E(G), \lambda_{l}(G-e)<k$. In this work we present a structural characterization of minimally $(k, k)$-edge-connected graph, for graphs without bridges. As a result, former characterization of minimally $(2,2)$-edge connected graph in [J. of Graph Theory, 3 (1979) 15-22] are extended. 


\section{ACKNOWLEDGMENTS}

I wish to express my gratitude to the faculty of the department of Mathematics at West Virginia University for many ways in which they have contributed to my mathematical career. Further, I want to thank my friends and fellow students for helping whenever I needed. My sincere thanks to an excellent advisor Professor Hong-Jian Lai for his outstanding and stimulating guidance and for his advice, encouragement, patience, and endurance during the time I was working on this dissertation and degree. Sharing his ability of formulating new research topics and his enthusiasm for

graph theory with me is an invaluable treasure. Also, I thank Professors Harvey Diamond, Harumi Hattori, and Cun-Quan Zhang of the Department of Mathematics and Professor Srinivas Kankanhalli of the Department of Computer Science, West Virginia University, for their time evaluating this dissertation and making constructive suggestions on improving it.

At a personal level, I thank my wife Saman Kumari Hennayake for her love and understanding, and look forward to our life together without the shadow of graduate school on either one of us. Our daughter Sanuri Tharaka has been a wonderfully cheerful presence and most worthwhile distraction during the last two years. 


\section{Contents}

1 Introduction 1

2 Notations and Terminology 3

3 Review of Literature $\quad 11$

3.1 Connectivity and edge-connectivity . . . . . . . . . . . . . . 11

3.1.1 Connectivity function . . . . . . . . . . . . . 14

3.2 Generalized connectivity and edge-connectivity . . . . . . . . . . . 14

3.2.1 l-connectivity function . . . . . . . . . . . . 20

3.3 Edge strength . . . . . . . . . . . . . . . . . 22

3.4 Minimally $(k, l)$-edge-connectivity . . . . . . . . . . . . 23

4 The lower bound of generalized edge-connectivity and optimal graphs 28

4.1 Lower bound of $\lambda_{l} \ldots \ldots \ldots \ldots$. . . . . . . . . . . . . . 28

4.2 Graphs reaching the lower bound with minimum number of edges . . 33

5 Relationship between generalized edge-connectivity and edge-strength 40

$5.1 \quad l$-sequential cuts . . . . . . . . . . . . . . . . . 41

5.2 Characterization of Extremal Graphs . . . . . . . . . . . . . . 48

6 Minimally $(k, k)$-edge connected graphs $\quad 53$ 
6.1 Elementary properties of minimally $(k, k)$-edge connected graphs . . . 53

6.2 Characterization of minimally $(k, k)$-edge connected graphs without

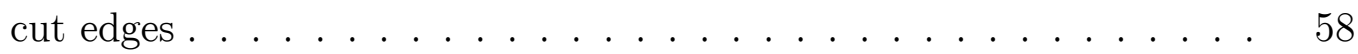

$\begin{array}{lll}7 & \text { Bibliography } & 64\end{array}$ 


\section{Chapter 1}

\section{Introduction}

Three problems are considered in this dissertation. They concern generalized edgeconnectivity of graphs and minimally edge-connected graphs. The first two problems deals with determining the lower bound and upper bound for the generalized edgeconnectivity, and the last problem is to find minimally $(k, k)$-edge connected graphs.

The basic notations and terminology used in this dissertation are given in Chapter 2. These notations and terminology are commonly found in most graph theory text books. Notations and definitions from Graphs and Digraphs by G. Chartrand and L. Lesniak [CL] are used for most of Chapter 2.

The review of Literature is given in Chapter 3. More definitions and notations are given in this chapter. These definitions and notations are not usually found in general graph theory text books. They may be found in some connectivity and edge-connectivity related advance text books and in journals related to connectivity, edge-connectivity, minimally connected graphs and minimally edge connected graphs. Notations and definitions from Explorations into graphs and connectivity by O.R. Oellermann [Oe4] are referenced in Chapter 3. 
Definitions and theorems related to connectivity and edge-connectivity are given in Section 3.1. Further edge-connectivity of some common simple graphs can also be found in this section which we will use in later chapters. In Section 3.2, definitions, theorems and some elementary properties of generalized connectivity and generalized edge-connectivity are given. Definition of edge strength and theorems of edge strength can be found in Section 3.3. Definitions and theorems related to minimally connected graphs and minimally edge-connected graphs can be found in Section 3.4.

For an integer $l>1$, the $l$-edge-connectivity of a graph with $|V| \geq l$, denoted by $\lambda_{l}$, is the smallest number of edges whose removal results in a graph with $l$ components. We consider the problem of finding the lower bound for the generalized edge-connectivity $\lambda_{l}$ in Chapter 4 . A lower bound for $\lambda_{l}$ using the edge-connectivity $\lambda$ is found. We prove that $\lambda_{l} \geq \frac{l}{2} \lambda$. Then we construct family of graphs satisfying the equality $\lambda_{l}=\frac{l}{2} \lambda$, and having a minimum number of edges. In the construction of these families of graphs we use circulant graphs.

In Chapter 5 an upper bound is found for the generalized edge-connectivity $\lambda_{l}$, using the edge strength $\bar{\lambda}$. We prove that $\lambda_{l} \leq(l-1) \bar{\lambda}$. A family of graphs satisfying the equality $\lambda_{l}=(l-1) \bar{\lambda}$ is constructed for the case $\lambda=\bar{\lambda}=2$.

Chapter 6 deals with minimally $(k, k)$-edge connected graphs without cut-edges. Several properties of minimally $(k, k)$-edge connected graphs are stated and proved. We present a structural characterization of minimally $(k, k)$-edge-connected graphs. As a result, former characterization of minimally $(2,2)$-edge-connected graph in [J. of Graph Theory, 3 (1979) 15-22] are extended. 


\section{Chapter 2}

\section{Notations and Terminology}

A simple graph $G$ is a finite non empty set of objects called vertices (the singular is vertex) together with a (possibly empty) set of unordered pairs of distinct vertices of $G$ called edges. The vertex set of $G$ is denoted by $V(G)$, while the edge set is denoted by $E(G)$. If one allows more than one edge (but yet a finite number) between the same pair of vertices in graph, the resulting structure is a multigraph. Such edges are called parallel edges. A loop is an edge that joins a vertex to itself. Unless otherwise mentioned, the graphs we consider are simple graphs.

The edge $e=\{u, v\}$ is said to join the vertices $u$ and $v$. If $e=\{u, v\}$ is an edge of a graph $G$, then $u$ and $v$ are adjacent vertices, while $u$ and $e$ are incident, as are $v$ and $e$. Furthermore, if $e_{1}$ and $e_{2}$ are distinct edges of $G$ incident with a common vertex, then $e_{1}$ and $e_{2}$ are adjacent edges. It is convenient to henceforth denote an edge by $u v$ or $v u$ rather than by $\{u, v\}$.

The cardinality of the vertex set of a graph $G$ is called the order of $G$ and is commonly denoted by $n(G)$, or more simply by $n$ when the graph under consideration is clear; while the cardinality of its edge set is the size of $G$ and is often denoted by 
$m(G)$ or $m$. An -graph has order $n$ and size $m$.

With the exception of the order and the size, the parameter that one encounters most frequently in the study of graphs is the degree of a vertex. The degree of a vertex $v$ in a graph $G$ is the number of edges of $G$ incident with $v$, which is denoted by $\operatorname{deg}_{G} v$ or simply by $\operatorname{deg} v$ if $G$ is clear from the context. A vertex is called even or odd according to whether its degree is even or odd. A vertex of degree 0 in $G$ is called an isolated vertex and a vertex of degree 1 is an end-vertex of $G$. The minimum degree of $G$ is the minimum among the vertices of $G$ and is denoted by $\delta(G)$. The maximum degree is defined similarly and is denoted by $\Delta(G)$. A graph has an associated degree list $d_{1}, d_{2}, \ldots, d_{n}$ where $d_{1} \leq d_{2} \leq \cdots \leq d_{n}$. Note that $d_{i}$ and $\operatorname{deg} v_{i}$ do not in general refer to the same vertex.

Two graphs often have the same structure, differing only in the way their vertices and edges are labeled or in the way they are drawn. To make this idea more precise, we introduce the concept of isomorphism. A graph $G_{1}$ is isomorphic to a graph $G_{2}$ if there exists a one-to-one mapping $\phi$, called an isomorphism, from $V\left(G_{1}\right)$ onto $V\left(G_{2}\right)$ such that $\phi$ preserves adjacency; that is $u v \in E\left(G_{1}\right)$ if and only if $\phi(u) \phi(v) \in E\left(G_{2}\right)$. It is easy to see that 'is isomorphic to' is an equivalence relation on graphs; hence, this relation divides the collection of all graphs into equivalence classes, two graphs being non isomorphic if they belong to different equivalence classes.

Frequently, a graph under study is contained within some larger graph also being investigated. We consider several instances of this now. A graph $H$ is a subgraph of a graph $G$ if $V(H) \subseteq V(G)$ and $E(H) \subseteq E(G)$; in such a case, we also say that $G$ 
is a supergraph of $H$. If $H$ is isomorphic to a subgraph of $G$, we also say that $H$ is a subgraph of $G$. If $H$ is a subgraph of $G$ then we write $H \subseteq G$.

Let $|X|$ denotes the cardinality of the set $X$. One type of subgraph of a graph $G$ is that obtained by deleting a vertex or edge. If $v \in V(G)$ and $|V(G)| \geq 2$, then $G-v$ denotes the subgraph with vertex set $V(G)-\{v\}$ and whose edges are all those of $G$ not incident with $v$; if $e \in E(G)$, then $G-e$ is the subgraph having vertex set $V(G)$ and edge set $E(G)-\{e\}$. The deletion of a set of vertices or set of edges is defined analogously.

If $u$ and $v$ are non adjacent vertices of a graph $G$, then $G+f$, where $f=u v$, denotes the graph with vertex set $V(G)$ and edge set $E(G) \cup\{f\}$, Clearly, $G \subseteq G+f$. We have seen that $G-e$ has the same vertex set as $G$ and that $G$ has the same vertex set as $G+f$. Whenever a subgraph $H$ of a graph $G$ has the same order as $G$, then $H$ is called a spanning subgraph of $G$.

A graph $G$ is regular of degree $r$ if $\operatorname{deg} v=r$ for each vertex $v$ of $G$. Such graphs are called $r$-regular. A graph is complete if every two of its vertices are adjacent. A complete $(n, m)$-graph is therefore a regular graph of degree $n-1$ having $m=n(n-1) / 2$; we denote this graph by $K_{n}$. A 3-regular graph is also called a cubic graph.

The complement $\bar{G}$ of a graph $G$ is that graph with vertex set $V(G)$ such that two vertices are adjacent in $\bar{G}$ if and only if these vertices are not adjacent in $G$. Hence, if $G$ is an $(n, m)$-graph, then $\bar{G}$ is an $(n, \bar{m})$ graph, where $m+\bar{m}=\left(\begin{array}{c}n \\ 2\end{array}\right)=\frac{n(n-1)}{2}$. The complement $\bar{K}_{n}$ of the complete graph $K_{n}$ has $n$ vertices and no edges and is 
referred to as the empty graph of order $n$.

A graph $G$ is bipartite, if it is possible to partition $V(G)$ into 2 subsets $V_{1}, V_{2}$ (called partite sets) such that every element of $E(G)$ joins a vertex of $V_{1}$ to a vertex of $V_{2}$. A complete bipartite graph $G$ is a bipartite graph with partite sets $V_{1}, V_{2}$ having the added property that if $u \in V_{1}$ and $v \in V_{2}$, then $u v \in E(G)$.

In the following definitions, we assume that $G_{1}$ and $G_{2}$ are two graphs with disjoint vertex sets. The union $G=G_{1} \cup G_{2}$ has $V(G)=V\left(G_{1}\right) \cup V\left(G_{2}\right)$ and $E(G)=E\left(G_{1}\right) \cup E\left(G_{2}\right)$. If a graph $G$ consists of $k(\geq 2)$ disjoint copies of a graph $H$, then we write $G=k H$. The join $G=G_{1}+G_{2}$ has $V(G)=V\left(G_{1}\right) \cup V\left(G_{2}\right)$ and $E(G)=E\left(G_{1}\right) \cup E\left(G_{2}\right) \cup\left\{u v \mid u \in V\left(G_{1}\right)\right.$ and $\left.v \in V\left(G_{2}\right)\right\}$. Using the join operation, we see that $K_{r, s}=\bar{K}_{r}+\bar{K}_{s}$.

Let $u$ and $v$ be (not necessarily distinct) vertices of a graph $G$. A $u-v$ walk of $G$ is a finite, alternating sequence

$$
u=u_{0}, e_{1}, u_{1}, e_{2}, \ldots, u_{k-1}, e_{k}, u_{k}=v
$$

of vertices and edges, beginning with vertex $u$ and ending with vertex $v$, such that $e_{i}=u_{i-1} u_{i}$ for $i=1,2, \ldots, k$. The number $k$ (the number of occurrences of edges) is called the length of the walk. A trivial walk contains no edges, that is $k=0$. We note that there may be repetition of vertices and edges in a walk. Often only the vertices of a walk are indicated since the edges present are then evident. Two $u-v$ walks $u=u_{0}, u_{1}, \ldots, u_{k}=v$ and $u=v_{0}, v_{1}, \ldots, v_{l}=v$ are considered to be equal if and only if $k=l$ and $u_{i}=v_{i}$ for $0 \leq i \leq k$; otherwise, they are different. Observe that the edges of two different $u-v$ walks of $G$ may very well induce the same subgraph of $G$. 
A $u-v$ walk is closed or open depending on whether $u=v$ or $u \neq v$. A $u-v$ trail is a $u-v$ walk in which no edge is repeated, while a $u-v$ path is a $u-v$ walk in which no vertex is repeated. Every path is therefore a trail. A vertex $u$ forms the trivial $u-u$ path.

A nontrivial closed trail of a graph $G$ is referred to as a circuit of $G$, and a circuit $v_{1}, v_{2}, \ldots, v_{n}, v_{1}(n \geq 3)$ whose vertices $v_{i}$ are distinct is called a cycle. An acyclic graph has no cycles. The subgraph of a graph $G$ induced by the edges of a trail, path, circuit or cycle is also referred to as a trail, path, circuit or cycle of $G$. A cycle is even if its length is even; otherwise it is odd. A cycle of length $n$ is an $n$-cycle; a 3-cycle is also called a triangle. A graph of order $n$ that is a path or a cycle is denoted by $P_{n}$ or $C_{n}$, respectively.

A set $S \subset V(G)$ is called an independent set of the graph $G$ if no two vertices in $S$ are adjacent in $G$. An independent set $S_{0}$ is a maximum independent set of $G$ if $\left|S_{0}\right| \geq|S|$, where $S$ is any independent set of $G$. The cardinality of a maximum independent set of a graph $G$ is called the independence number of $G$ and is denoted by $\beta(G)$.

We now consider a very basic concept in graph theory, namely connected and disconnected graphs. A vertex $u$ is said to be connected to a vertex $v$ in a graph $G$ if there exists a $u-v$ path in $G$. A graph $G$ is connected if every two of its vertices are connected. A graph that is not connected is disconnected. The relation 'is connected to' is an equivalence relation on the vertex set of every graph $G$. Each subgraph induced by the vertices in a resulting equivalence class is called 
a connected component or simply a component of $G$. Equivalently, a component of a graph $G$ is a connected subgraph of $G$ not properly contained in any other connected subgraph of $G$; that is, a component of $G$ is a subgraph that is maximal with respect to the property of being connected. Hence, a connected subgraph $F$ of a graph $G$ is a component of $G$ if for each connected graph $H$ with $F \subseteq H \subseteq G$ where $V(F) \subseteq V(H)$ and $E(F) \subseteq E(H)$, it follows that $F=H$. The number of components of $G$ is denoted by $k(G)$; of course, $k(G)=1$ if and only if $G$ is connected.

Although being connected is the most basic structural property that a graph may posses, more information about its structure is provided by special vertices, edges and subgraphs it contains and the symmetry it possesses.

Some graphs are connected so slightly that they can be disconnected by the removal of a single vertex or single edge. Such vertices and edges play a special role in graph theory.

A vertex $v$ of a graph $G$ is called a cut-vertex of $G$ if $k(G-v)>k(G)$. Thus, a vertex of connected graph is a cut-vertex if its removal produces a disconnected graph. In general, a vertex $v$ of a graph $G$ is a cut-vertex of $G$ if its removal disconnects a component of $G$.

Analogous to the cut-vertex is the concept of a bridge. A bridge or cut-edge of a graph $G$ is an edge $e$ such that $k(G-e)>k(G)$. If $e$ is a cut-edge of $G$, then it is immediately evident that $k(G-e)=k(G)+1$. Furthermore, if $e=u v$, then $u$ is a cut-vertex of $G$ if and only if $\operatorname{deg} u>1$. 
Thus if $v$ is a cut-vertex of a connected graph $G$, then $G-v$ is disconnected. A nonseparable graph is connected nontrivial, and has no cut-vertices. A block of a graph is a maximal nonseparable subgraph. If $G$ is nonseparable, then $G$ itself is often called a block.

A tree is an acyclic connected graph, while a forest is an acyclic graph. Thus every component of a forest is a tree. A spanning tree of a graph $G$ is a spanning subgraph of $G$ that is a tree. Every connected graph $G$ contains a spanning tree. If $G$ is itself a tree, then this observation is trivial. If $G$ is not a tree, then a spanning tree $T$ of $G$ can be obtained by removing cycle edges from $G$ one at a time until only bridges remain. If $G$ has order $n$ and size $m$, then since $T$ has size $n-1$, it is necessary to delete a total of $m-(n-1)=m-n+1$ edges to produce $T$. This of course, implies that $m \geq n-1$, that is, every connected graph of order $n$ has at least $n-1$ edges.

A vertex-cut in a graph $G$ is a set $U$ of vertices of $G$ such that $G-U$ is disconnected. Every graph that is not complete has a vertex-cut. Indeed, the set of all vertices distinct from two non adjacent vertices is a vertex-cut. Of course, the removal of any proper subset of vertices from a complete graph leaves another complete graph. The vertex-connectivity or simply the connectivity $\kappa(G)$ of a graph $G$ is the minimum cardinality of a vertex-cut of $G$ if $G$ is not complete, and $\kappa(G)=n-1$ if $G=K_{n}$ for some $n$. Hence $\kappa(G)$ is the minimum number of vertices whose removal results in a disconnected or trivial graph. It is an immediate consequence of the definition that a nontrivial graph has connectivity 0 if and only if $G$ is disconnected. Furthermore, a graph $G$ has connectivity 1 if and only if $G=K_{2}$ or $G$ is a connected graph with cut-vertices; $\kappa(G) \geq 2$ if and only if $G$ is non separable of order 3 or more. 
Connectivity has an edge analogue. An edge-cut in a graph $G$ is a set $X$ of edges of $G$ such that $G-X$ is disconnected. If $X$ is a minimal edge-cut of a connected graph $G$, then, necessarily, $G-X$ contains exactly two components. Every nontrivial graph has an edge-cut. The edge-connectivity $\lambda(G)$ of a graph $G$ is the minimum cardinality of an edge-cut if $G$ is nontrivial, and $\lambda\left(K_{1}\right)=0$. So $\lambda(G)$ is the minimum number of edges whose removal from $G$ results in a disconnected or trivial graph. Thus $\lambda(G)=0$ if and only if $G$ is disconnected or trivial; while $\lambda(G)=1$ if and only if $G$ is connected and contains a bridge.

Throughout this paper we assume $\lambda(G)>1$. For disjoint non empty subsets $A, B \subset V(G)$, the set $[A, B]$ denotes all edges in $G$ with one vertex in $A$, and the other in $B$. We also use the notations $\lceil x\rceil$ to denote the smallest integer greater than or equal to $x$, and $\lfloor x\rfloor$ for the largest integer less than or equal to $x$.

Let $G$ be a graph and let $X \subset E(G)$ be an edge subset. The contraction $G / X$ is the graph obtained from $G$ by identifying the two ends of each edge in $X$ and by deleting the resulting loops. Thus $G / X$ is loopless and may have multiple edges, even when $G$ is simple. If $H$ is a subgraph of $G$, then $G / H$ denotes $G / E(H)$. Note that each vertex $v$ in $G / X$ is the contraction image of a connected subgraph $H_{v}$ of $G$. Thus $H_{v}$ is called the preimage of $v$. A vertex $v$ in the contraction $G / X$ is nontrivial if $\left|V\left(H_{v}\right)\right|>1$. 


\section{Chapter 3}

\section{Review of Literature}

Every graph can be thought of as a model for a network, such as a road network, an electrical network or a communication network. Many attempts have been made to determine how well such a network is "connected" or stated differently how much effort is required to break down communication in the system.

\subsection{Connectivity and edge-connectivity}

Two very well-known measures that indicate how "reliable" a graph is, are the "connectivity" and "edge-connectivity" of a graph. The complete graph $K_{n}$ on $n$ vertices has both connectivity and edge-connectivity equal to $n-1$, while the complete bipartite graph $K_{i, j}$, where $i \leq j$, has both connectivity and edge-connectivity equal to $i$. The graph $G$ of Figure 3.1 shows that these two parameters are not equal, since $\kappa(G)=2$ and $\lambda(G)=3$. A classic result of Whitney's [Wh1] states however, that the connectivity of a graph never exceeds its edge-connectivity. Moreover, if $\delta(G)$ is the minimum degree of $G$, then it is not difficult to see that $\delta(G)$ is an upper bound for the edge-connectivity of $G$. Consequently, we have the following fundamental relationship between the connectivity, edge-connectivity and minimum degree of a graph. 
Theorem 3.1 (Whitney [Wh1], 1932) For any graph $G$,

$$
\kappa(G) \leq \lambda(G) \leq \delta(G)
$$

Chartrand and Harary $[\mathrm{CH}]$ constructed a family of graphs with prescribed connectivities which also have a minimum degree. This result shows that the restrictions on $\kappa, \lambda$, and $\delta$ imposed by Theorem 3.1 cannot be improved.

Chartrand pointed out that if $\delta$ is large enough, then the second inequality of Theorem 3.1 becomes an equality.

Theorem 3.2 (Chartrand [Cha], 1966) If $G$ is a graph with order $n$, and $\delta(G) \geq n / 2$, then $\lambda(G)=\delta(G)$.

For example if $G$ is regular of degree $r \geq n / 2$, then $\lambda(G)=r$. In particular, $\lambda\left(K_{n}\right)=n-1$.

The analogue of Theorem 3.2 for connectivity does not hold. The problem of determining the largest connectivity possible for a graph with a given number of vertices and edges was proposed by Berge [Ber] (1958) and a solution was given by Harary.

Theorem 3.3 (Harary [Har] 1962) Among all (n,m)-graphs, the maximum connectivity is 0 when $m<n-1$ and is $\left\lceil\frac{2 m}{n}\right\rceil$, when $m \geq n-1$.

To show that this value can actually be attained, an appropriate family of graphs can be constructed. These graphs are called Harary graphs. However, an interesting subclass of symmetric graphs known as circulants generalize the Harary graphs. They 
can be defined as follows. Let $|V(G)|=n$ be a positive integer. Assume that the vertices of a graph are labeled $0,1,2, \ldots, n-1$, and we refer to vertex $i$ instead of saying the vertex labeled $i$. The circulant graph $C_{n}\left[a_{1}, a_{2}, \ldots, a_{k}\right]$ or briefly $C_{n}\left[a_{i}\right]$, where $0<a_{1}<a_{2}<\cdots<a_{k}<\frac{n+1}{2}$, has $i \pm a_{1}, i \pm a_{2}, \ldots, i \pm a_{k}(\bmod n)$ adjacent to each vertex $i$. The sequence $\left\langle a_{i}\right\rangle$ is called the jump sequence and the $a_{i}$ 's are called the jumps. Notice that our definition precludes jumps of size greater than $\frac{n}{2}$ as such jumps would produce the same result as a jump of size $(n-a)$, where $n-a<\frac{n}{2}$. Also note that if $a_{k} \neq \frac{n}{2}$ then the circulant is always regular of degree $2 k$. The circulant

graph $C_{5}[1,2]$ is given in Figure 3.2. When $n$ is even we have allowed $a_{k}=\frac{n}{2}$ (called a diagonal jump), and when $a_{k}=\frac{n}{2}$ the circulant has degree $2 k-1$. The circulant graph $C_{6}[1,3]$ is given in Figure 3.3. We use circulant graphs in Chapter 4. Circulant graphs also gives those $(n, m)$-graphs with maximum edge-connectivity.

Corollary 3.3(a) The maximum edge-connectivity of a $(n, m)$-graph equals the maximum connectivity.

Below we state some results of edge-connectivity. Some of these results are used in Chapter 4.

\section{Edge-connectivity of some graphs:}

1. $\lambda(T)=1$.

2. $\lambda\left(C_{n}\right)=2$.

3. $\lambda\left(K_{n}\right)=n-1$, for the complete graph $K_{n}$ of order $n$.

4. $\lambda\left(K_{\left(n_{1}, n_{2}\right)}\right)=n_{1}$, for the complete bipartite graph $K_{\left(n_{1}, n_{2}\right)}, n_{1} \leq n_{2}$. 
5. For the circulant graph $C_{n}[1,2, \ldots, i]$, with $i<\frac{n+1}{2}$ :

$$
\lambda\left(C_{n}[1,2, \ldots, i]\right)=\left\{\begin{array}{cc}
2 i & \text { for } i<\frac{n}{2} \\
2 i-1 & \text { for } i=\frac{n}{2}
\end{array}\right.
$$

\subsubsection{Connectivity function}

Beineke and Harary $[\mathrm{BH}]$ referred to a pair $(s, t)$ of non negative integers as a connectivity pair for distinct vertices $u$ and $v$ of a graph $G$ if there is a set of $s$ vertices and $t$ edges whose removal separates $u$ and $v$, but no set of $s-1$ vertices and $t$ edges or $s$ vertices and $t-1$ edges has this property. Similarly, a pair $(s, t)$ of non negative integers is a connectivity pair for a non complete graph $G$ if there exist $s$ vertices and $t$ edges whose removal disconnects $G$ but no set of $s-1$ vertices and $t$ edges or $s$ vertices and $t-1$ edges has this property. For each integer $s(0 \leq s \leq \kappa(G))$, there is a unique connectivity pair $\left(s, t_{s}\right)$; thus $G$ has exactly $\kappa(G)+1$ connectivity pairs. This observation motivated Beineke and Harary to define the connectivity function $f$ of a graph $G$, where $f$ maps the set $\{0,1, \ldots, \kappa(G)\}$ into the non negative integers such that $f(s)=t_{s}$ for $0 \leq s \leq \kappa(G)$. Hence $f(\kappa(G))=0$. They further observed that the connectivity function of a graph $G$ is strictly decreasing.

\subsection{Generalized connectivity and edge-connectivity}

Thus far, main interest has been to determine a smallest set $S$ of vertices or of edges or of a combination of vertices and edges in a graph $G$ such that $G-S$ is disconnected or trivial, without regard to the number of components of $G-S$. Two graphs with the same connectivity may have differing degrees of vulnerability in the sense that the deletion of a vertex cutset of minimum cardinality from the one graph may produce a graph with considerably more components, than in the case of the other graph. For example, star $K_{1, n}$ and the path $P_{n+1}(n \geq 2)$ are both trees of order 
$n+1$ and therefore have connectivity 1 , but the deletion of a cut-vertex from $K_{1, n}$ produces $n$ components, while the deletion of a cut-vertex from $P_{n+1}$ produces only two components.

These observations might have motivated Chartrand, Kapoor, Lesniak and Lick [CKLL] (1984), to define for an integer $l \geq 2$ and a graph $G$ of order $n \geq l$ the $l$ connectivity $\kappa_{l}(G)$ to be the smallest number of vertices whose removal from $G$ results in a graph with at least $l$ components or a graph of order less than $l$. If $l=2$, then $\kappa_{l}(G)=\kappa(G)$. The l-connectivity of a graph is thus a generalization of the connectivity of a graph. Moreover, the maximum $l$ such that $\kappa_{l}(G)=\kappa(G)$ is the maximum number of components that are produced when a vertex cutset of minimum number of cardinality is deleted from $G$.

If $G$ is a graph of order $n$ for some integer $n \geq 1$ and $l$ is an integer with $2 \leq l \leq n$, then the l-edge connectivity $\lambda_{l}(G)$ of $G$ is the minimum number of edges that need to be deleted from $G$ to produce a graph with at least $l$ components. Generalized edge connectivity was first defined by Boesch and Chen [BoC] (1978). In order to be consistent with $\lambda$ let $\lambda_{2}\left(K_{1}\right)=0$. Hence, if $l=2$, then $\lambda_{l}(G)=\lambda(G)$. In Figure 3.4, we see that $\lambda_{2}=1, \lambda_{3}=2, \lambda_{4}=3$.

It seems natural to seek an extension for Whitney's result in Theorem 1, that is, one may investigate whether $\kappa_{l}(G) \leq \lambda_{l}(G)$ is true for $l \geq 3$. However, it turns out that this proposed inequality fails even for $l=3$. Consider, for example, the graph $G$ of Figure 3.5. For this graph $\lambda_{3}(G)=2$ while $\kappa_{3}(G)=4$. In fact, Chartrand, Kapoor, Lesniak and Lick [CKLL] established the following much stronger result. 
Theorem 3.4 (Chartrand, Kapoor, Lesniak and Lick [CKLL], 1984) Let $l \geq 3$ be an integer and let $i, j$ be positive integers such that $i \geq l-1$ and $j \geq 1$. Then there is a graph $G$ such that $\kappa_{l}(G)=j$ and $\lambda_{l}(G)=i$.

Sampathkumar [Sam] showed, however, that under certain conditions Theorem 3.1 can be generalized.

Theorem 3.5 (Sampathkumar [Sam], 1984) Let $G$ be a graph without isolated vertices, order $n$ independence number $\beta(G)$ and $c$ components. If $d_{1} \leq d_{2} \leq \cdots \leq d_{n}$ is the degree sequence of $G$ and $2 \leq l \beta(G)$, then $\kappa_{l}(G) \leq \lambda_{l}(G) \leq D$, where $D=0$ if $l \leq c$ and $\sum_{i=1}^{l-1} d_{i}$ if $l>c$.

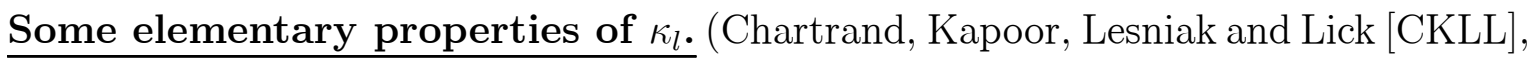
1984)

1. If the (vertex) independent number $\beta(G)$ of $G$ is less than $l$, then $\kappa_{l}(G)=n-l+1$.

2. If $a \leq b \leq \beta(G)$, then $\kappa_{2}(G) \leq \kappa_{b}(G)$ of $G$.

3. $\kappa_{l}(G)=0$ if $G$ is empty graph $\bar{K}_{n}$ of order $n$, and $\kappa_{n}(G)=1$ otherwise.

4. $\kappa_{n-1}(G)=0,1$ or 2 .

(If $\beta(G)=n$, then $\kappa_{n-1}(G)=0$; if $\beta(G)=n-1$, then $\kappa_{n-1}(G)$ is 0 or 1 ; for $\beta(G)<n-1, \kappa_{n-1}(G)=2$.)

Examples: (Chartrand, Kapoor, Lesniak and Lick [CKLL], 1984)

1. $\kappa_{l}(T)=l-1$.

2. $\kappa_{l}\left(C_{n}\right)=l$.

3. $\kappa_{l}\left(K_{n}\right)=n-l+1$ for the complete graph $K_{n}$ of order $n$. 
4. For the complete bipartite graph $K_{\left(n_{1}, n_{2}\right)}, n_{1} \leq n_{2}$ :

$$
\kappa_{l}\left(K_{\left(n_{1}, n_{2}\right)}\right)=\left\{\begin{array}{cc}
n_{1} & \text { if } n_{2} \geq l \geq 2 \\
n_{1}+n_{2}-l+1 & \text { if } n_{2}<l .
\end{array}\right.
$$

For a graph $G$ of order $n$ the sequence $s: \kappa_{2}(G), \kappa_{3}(G), \ldots, \kappa_{n}(G)$ is called the connectivity sequence. Chartrand, Kapoor, Lesniak and Lick [CKLL] characterized l-connectivity sequences in the following theorem.

Theorem 3.6 (Chartrand, Kapoor, Lesniak and Lick [CKLL], 1984) A sequence $k_{2}, k_{3}, \ldots, k_{n}$ of nonnegative integers is realized as the connectivity sequence $\kappa_{2}(G), \kappa_{3}(G), \ldots, \kappa_{n}(G)$ of a graph $G$ of order $n$ if and only if there exists an integer $j$ such that $k_{2} \leq k_{3} \leq$ $\ldots \leq k_{j} \leq k_{j+1}$ and $k_{j+i}=n-(j+i)+1$ for $i=1,2, \ldots, n-j$. Moreover, $j=\beta(G)$ is the independence number of $G$.

Theorem 3.7 (O.R. Oellermann [Oe3], 1987) Let $G$ be a graph of order $n \geq 2$ the degrees $d_{i}$ of whose vertices satisfy $d_{1} \leq d_{2} \leq \cdots \leq d_{n}$. Suppose $k$ and $l \geq 2$ are integers with $1 \leq k \leq n-l+1$. If $d_{j} \leq j+k-2 \Rightarrow d_{n-k+1} \geq n-j(l-1)$ for each $j$ such that $1 \leq l \leq\lfloor(n-k+1) / l\rfloor$, then $\kappa_{l}(G) \geq k$.

Some elementary properties of $\lambda_{l}$. (F.T. Boesch and S. Chen [BoC], 1978)

1. $\lambda_{l}$ exists for each $l$ in the range $2 \leq l \leq n$; where $|V|=n$.

2. If $Y$ is an $l$-cut of size $\lambda_{l}$, then no edge of $Y$ has both of its endpoints in the same component of $G-Y$.

3. When $G$ is connected $\lambda_{l}(G-e)+1 \leq \lambda_{l}(G)$ for some edge $e \in E$, and $\lambda_{l}(G-e)+1 \geq$ $\lambda_{l}(G)$ for all edges $e \in E$.

4. For an $(n, m)$-graph $\lambda_{n}=m$. 
Examples: (F.T. Boesch and S. Chen [BoC], 1978)

1. $\lambda_{l}(T)=l-1$.

2. $\lambda_{l}\left(C_{n}\right)=l$.

3. $\lambda_{l}\left(K_{n}\right)=\frac{(l-1)}{2}(2 n-l)$ for the complete graph $K_{n}$ of order $n$.

It is easy to see that for any tree $T, \lambda_{l}(T)=l-1$. Furthermore any connected graph $G$ contains a tree spanning all the vertices of $G$. Hence $\lambda_{l}(G) \geq l-1$ for connected graphs.

It should be noted that a number of authors have raised the question of finding algorithms to determine the value of $\lambda_{l}$. For example, Hu [HU] lists this analysis problem as an "open question." Solution of this can be found in [Ma5].

The following three lower bounds for generalized edge-connectivity $\lambda_{l}$ were given by F.T. Boesch and S. Chen [BoC], in 1978.

Theorem 3.8 (F.T. Boesch and S. Chen $[\mathrm{BoC}], 1978$ ) For connected graphs with $1 \leq i<l-1<n$,

$$
\lambda_{l} \geq \frac{(l-1)(l-i+1)}{(l+1)(l-i-1)} \lambda_{l-i} .
$$

Theorem 3.9 (F.T. Boesch and S. Chen $[\mathrm{BoC}]$, 1978) Let $G$ be a graph with $1<l \leq n$ and minimum degree $\delta$. If $\delta \geq\left\lfloor\frac{n}{l}\right\rfloor$ then $\lambda_{l} \geq \delta$.

Next theorem gives bounds on $\lambda_{l}$ given the information on the degrees of the graph. They are useful because the calculation of $\lambda_{l}$ can be tedious, whereas the 
degree sequence is probably the most readily available data concerning the structure of a graph.

Theorem 3.10 (F.T. Boesch and S. Chen [BoC], 1978) Let $G$ be a graph with $1<l \leq$ $n$ and degree list $d_{1} \leq d_{2} \leq \cdots \leq d_{n}$. If $d_{l} \geq\left\lfloor\frac{(l-1) n}{l}\right\rfloor$ then $\lambda_{l} \geq\left(\sum_{i=1}^{l-1} d_{i}\right)-\frac{(l-1)(l-2)}{2}$.

D.L. Goldsmith, B Manvel and V. Faber [GMF] defined $\sigma_{3}(G)=\min \left\{|S|+\lambda^{\prime}(G-\right.$ $S)\}$, where the minimum is taken over all sets $S$ of $\lambda(G)$ edges whose removal separates the connected graph $G$ into two components. Here $\lambda^{\prime}(G-S)$ gives the minimum number of edges whose removal incerases the number of components by one. They showed that $\lambda_{3}=\sigma_{3}$ if $\sigma_{3}$ is sufficiently large.

Theorem 3.11 (D.L. Goldsmith, B Manvel and V. Faber [GMF], 1980) Let $G$ be a connected graph of order $n \geq 3$. If

$$
\sigma_{3}(G) \geq \begin{cases}n-2, & \text { for } n \text { even } \\ n-1, & \text { for } n \text { odd }\end{cases}
$$

then $\lambda_{3}(G)=\sigma_{3}(G)$.

D.L. Goldsmith showed that if the degrees of the vertices are sufficiently large, then either $\lambda_{3}=\sigma_{3}$ or else every $\lambda_{3}$ set consists of the set of all edges incident with a single vertex $v$, together with a set of at most $\lambda(G)-1$ edges in $G-v$.

Theorem 3.12 (D.L. Goldsmith [Go1], 1980) Let $G$ be a connected graph with $n \geq 3$ vertices. If $\sigma_{3}(G) \geq 2\left[\frac{n}{3}\right]$, then either

(1) $\lambda_{3}(G)=\sigma_{3}(G)$, or

(2) every $\lambda_{3}(G)$ set of $G$ consists of 
(a) all edges incident with a single vertex $v$ of degree $>\lambda(G)$, plus

(b) a set of $<\lambda(G)$ edges in $G-v$.

Moreover, the lower bound $2\left[\frac{n}{3}\right]$ for $\sigma_{3}(G)$ is best possible.

Following sufficient conditions for $\lambda_{l} \geq \delta$ were given by Goldsmith [Go2] in 1981.

Theorem 3.13 (Goldsmith [Go2] 1981) If $l(\delta+1)>n$ then $\lambda_{l} \geq \delta$.

For the next two theorem, let the degree sequence of the vertices of $G$ is given by $\delta=d_{1} \leq d_{2} \leq \cdots \leq d_{n}$. For $1 \leq j \leq n, \delta^{(j)}=\sum_{i=1}^{j} d_{i}$.

Theorem 3.14 (Goldsmith [Go2] 1981) Suppose $l \leq \delta$ and $l(\delta+1) \leq n$. If $\frac{\delta^{(2 l)}}{2 l}>$ $\frac{n}{l}-1$, then $\lambda_{l} \geq \delta$. Moreover, the inequality cannot be replace by an equality.

Theorem 3.15 (Goldsmith [Go2] 1981) Suppose $l \leq \delta$ and $l(\delta+1) \leq n$. If $\frac{\delta^{(a)}}{a}>$ $\frac{n}{l}-1+\frac{2(\delta-1)}{l(\delta+1)}$ for $a=l(\delta+1)$, then $\lambda_{l} \geq \delta$.

\subsection{1 l-connectivity function}

When a minimum separating set of vertices, edges or both vertices and edges is removed from a graph, then, by definition, a disconnected graph results. However, some resulting graphs contain more components than others. The notions of the connectivity pairs and the corresponding connectivity function of a graph may be generalized in a natural way. Suppose that $G$ is a graph and $l \geq 2$ a fixed integer. For $k \in\left\{0,1, \ldots, \kappa_{l}(G)\right\}$, let $s_{k}$ be the minimum $l$-edge connectivity among all subgraphs obtained by removing $k$ vertices from $G$. Then $\left(k, s_{k}\right)$ is defined to be an 
l-connectivity pair of $G$. Hence every graph $G$ has $\kappa_{l}(G)+1 l$-connectivity pairs.

For a given graph $G$ and integer $k \in\left\{0,1, \ldots, \kappa_{l}(G)\right\}$ there is a unique $l$-connectivity pair $\left(k, s_{k}\right)$ of $G$. Consequently, the $l$-connectivity pairs of $G$ determine a function $f_{l}$, called the l-connectivity function of $G$, having $\left\{0,1, \ldots, \kappa_{l}(G)\right\}$ as its domain and the nonnegative integers as its codomain such that $f_{l}(k)=s_{k}$ for $0 \leq k \leq \kappa_{l}(G)$. As an example, consider the $l$-connectivity function of complete graphs. Let $l, n \geq 2$ be integers. If $n<l$, then $\kappa_{l}\left(K_{n}\right)=0$ so that $f_{l}$ has domain and range $\{0\}$. For $n \geq l$, O.R. Oellermann [Oe2] proved the following results.

Theorem 3.16 (O.R. Oellermann [Oe2], 1987) Let $n, l \geq 2$ be integers with $n \geq l$. Then the $l$-connectivity function of $K_{n}$ is given by

$$
f_{l}(k)=\left\{\begin{array}{cc}
(l-1)(n-l-k-1)+\left(\begin{array}{c}
l-1 \\
2
\end{array}\right) & 0 \leq k \leq \kappa_{l}(G) \\
0 & k=\kappa_{l}(G)
\end{array}\right.
$$

Theorem 3.17 (O.R. Oellermann [Oe2], 1987) For a graph $G$ and integer $l \geq 3$, the $l$-connectivity function $f_{l}$ of $G$ is nonincreasing. Further, $f_{l}\left(\kappa_{l}(G)\right)=0$ while $f_{l}(k)>0$ for $0 \leq k<\kappa_{l}(G)$.

Theorem 3.18 (D.P. Day, O.R. Oellermann and H.C. Swart [DOS], 1991) Suppose $G \cong K_{m_{1}, m_{2}, \ldots, m_{j}}$ where $m_{1} \leq m_{2} \leq \cdots \leq m_{j}$ and $j \geq 2$. Let $n=\sum_{i=1}^{j} m_{i}$ and let $k$ be an integer with $0 \leq k \leq \kappa_{l}(G)$. If $s=\min \left\{m_{j-1}, \sum_{i=1}^{j-1} m_{i}-k\right\}$, then the 
l-connectivity function of $G$ is given by

$$
f_{l}(k)=\left\{\begin{array}{cc}
0 & \text { if } k=\kappa_{l}(G) \\
(l-1)\left(n-m_{j}-k\right) & \text { if } k \neq \kappa_{l}(G) \text { and } l \leq m_{j}-s+2 \\
(l-1)\left(n-m_{j}-k\right)-\left(\begin{array}{c}
l-m_{j}+s-1 \\
2
\end{array}\right) & \text { if } k \neq \kappa_{l}(G) \text { and } l>m_{j}-s+2 .
\end{array}\right.
$$

The l-edge-connectivity has been studied extensively in [BoC, Go2, Go3] and [Sam]. An excellent surveys on explorations into graph connectivity was written by Oellermann [Oe4] and on theoretical aspects of connectivity in graphs and digraphs was written by Mader [Mad3].

\subsection{Edge strength}

Many graph theoretic problems can be decomposed and simplified by treating the problem separately on the components and/or blocks of the graph, and it is of interest to consider other subgraphs of a graph characterized by their connectivities over which certain graph theoretic questions may be decomposed and resolved, It is generally geometrically evident that the intensity of connectivity can vary widely over

different parts of a graph, and in order to determine special subgraphs characterized by their connectivities it is expedient to have a measure of the local intensity of connectivity within a graph.

The edge strength or the strength $\bar{\lambda}(G)$, of $G$ was introduced by Matula [Ma2]. For any subgraph $H$ of graph $G$,

$$
\bar{\lambda}(G)=\max \{\lambda(H): H \text { is a subgraph of } G\} .
$$

The invariant $\bar{\lambda}(G)$, has been studied in [BM, Ma2, Lai], among others. We use the 
strength to find an upper bound for $\lambda_{l}$.

The ordered partition of the edges of the graph $G, Z=\left(X_{1}, X_{2}, \ldots, X_{l}\right)$, is a slicing of $G$ if

$$
X_{i} \text { is a cut set of }\left\{\begin{array}{cc}
G & \text { for } i=1 \\
G-\cup_{j=1}^{i-1} X_{j} & \text { for } 2 \leq i \leq m
\end{array}\right.
$$

and each $X_{i}$ will also be termed a cut of the slicing. D.W. Matula [Ma2] main concern with slicings is not in the number of cuts but rather in the number of edges in the biggest cut of the slicing. Thus, the width $w(Z)$ of the slicing $Z$ of $G$ is defined by $w(Z)=\max \{|X|: X$ is a cut of the slicing $Z\}$. The duality between slicings and strength in a graph is evident in the following min-max theorem.

Theorem 3.19 (D.W. Matula [Ma2], 1969) For any graph $G$ containing at least one edge, the minimum width of any slicing of $G$ is equal to the strength of the graph, that is, $\min \{w(Z): Z$ is a slicing of $G\}=\max \{\lambda(H): H$ is a subgraph of $G\}$. A simple graph $G$ is $k$-maximal if $|V(G)|>k, \bar{\lambda}(G) \leq k$ and if for any edge $e$ of $\bar{G}, \bar{\lambda}(G+e)>k$.

Theorem 3.20 (H.J. Lai [Lai], 1990) If $n=|V(G)|>k+1$ and $G$ is a $k$-maximal graph, then $\bar{\lambda}(G)=\lambda(G)=k$.

\subsection{Minimally $(k, l)$-edge-connectivity}

R. Halin proved in 1969 [Hal], that every $n$-connected finite graph $G$ has a vertex of degree $n$ or an edge $e$ such that the graph $G-e$ which arises from $G$ by deleting $e$ is still $n$-connected. This theorem was the stating point for an extensive literature. A graph $G$ is minimally $k$-connected if $\kappa(G) \geq k$ and $\kappa(G-e)<k$ for every edge $e$ of $G$. Analogously a graph $G$ is minimally $k$-edge-connected if $\lambda(G) \geq k$ and $\lambda(G-e)<k$ 
for every edge $e$ of $G$. Further, a graph $G$ is critically $k$-connected if $\kappa(G) \geq k$ and $\kappa(G-v)<k$ for every vertex $v$ of $G$. A graph $G$ is critically $k$-edge-connected if $\lambda(G) \geq k$ and $\lambda(G-v)<k$ for every vertex $v$ of $G$. Rich theories of these concepts have been developed (see, for example, [Lic] and [Mad3].)

Since the 1-edge connected graphs are simply the nontrivial connected graphs and every nontrivial connected graph $G$ has at least two vertices $u$ and $v$ such that $G-u$ and $G-v$ are connected, it follows that the only critically 1-edge connected graph is the complete graph with two points, $K_{2}$. It is easily observed that a graph is minimally 1-edge connected if and only if it is a tree; thus if $G$ is a graph with $\lambda(G)=1$ and $\delta(G) \geq 2$, then $G$ is neither critically 1-edge connected nor minimally 1-edge connected.

The concept of "critically $k$-edge connected" and "minimally $k$-edge connected" are independent in the sense that neither property implies the other. For example, the graph $G_{2}$ of Figure 3.6 is critically 2-edge connected but not minimally 2-edge connected, while the graph $G_{1}=K_{2,3}$ of Figure 3.7 is minimally 2-edge connected, but not critically 2-edge connected.

Theorem 3.21 (Lick [Lic] 1970) Every graph $G$ with $\lambda(G)=k$ contains a critically $k$-edge connected subgraph and a minimally $k$-edge connected subgraph.

Occasionally, we are interested in determining whether the connectivity or edgeconnectivity of a graph is at least $k$, for some non-negative integer $k$, rather than finding the parameters exactly. With this in mind we say that a graph $G$ is $k$-connected if $\kappa(G) \geq k$. Thus a graph $G$ is $k$-connected for every $k$ satisfying $0 \leq k \leq \kappa(G)$. Sim- 
ilarly graph $G$ is $k$-edge-connected if $\lambda(G) \geq k$. Thus a graph $G$ is $k$-edge-connected for every $k$ satisfying $0 \leq k \leq \lambda(G)$. We note that a nontrivial graph is 1 -connected if and only if it is connected, and that it is 2-connected if and only if it is a block having more than one edge.

The concept of $k$-connectedness in a graph may be extended as follows. A graph $G$ is $(k, l)$-connected if $\kappa_{l}(G) \geq k$. Consequently, a graph is $(k, 2)$-connected if and only if it is $k$-connected. Analogously a graph $G$ is $(k, l)$-edge-connected if $\lambda_{l}(G) \geq k$. Consequently, a graph is $(k, 2)$-edge-connected if and only if it is $k$-connected.

The concept of minimally $k$-connected and minimally $k$-edge-connected in a graph may be extended as follows. A graph $G$ is minimally $(k, l)$-connected if $\kappa_{l}(G) \geq k$ and $\kappa_{l}(G-e)<k$ for every edge $e$ of $G$. Thus, a graph is minimally $(k, 2)$-connected if and only if it is minimally $k$-connected. Analogously a graph $G$ is minimally $(k, l)$ edge-connected if $\lambda_{l}(G) \geq k$ and $\lambda_{l}(G-e)<k$ for every edge $e$ of $G$. Thus, a graph is minimally $(k, 2)$-edge-connected if and only if it is minimally $k$-edge connected. Minimally $(2,2)$-connected graphs have been characterized by Dirac [Dir] and Plummer [Plu]. Minimally $(2,2)$-edge connected graphs have been characterized by Chaty and Chein $[\mathrm{CC}]$. Chaty and Chein showed that the set of nonseparable minimally $(2,2)$ edge connected graphs is strictly included in the set of minimally $(2,2)$-connected graphs. The graph of Figure 3.8 is minimally $(2,2)$-connected but not minimally $(2,2)$-edge connected.

Theorem 3.22 (W. Mader [Mad1], 1971) Every minimally $(k, 2)$-edge connected, finite graph has at least $k+1$ vertices of degree $k$. 
In [CKLL] the following sufficient condition for a graph to be $(k, l)$-connected is established.

Theorem 3.23 (Chartrand, Kapoor, Lesniak and Lick [CKLL], 1984) Let $G$ be a graph of order $n$ with $\beta(G) \geq l$. If the minimum degree $\delta(G)$ satisfies $\delta(G) \geq$ $\frac{n+(l-1)(k-2)}{l}$, then $G$ is $(k, l)$-connected.

A necklace is a nonseparable minimally 2-edge connected simple graph. Therefore a nonseparable minimally 2-edge connected graph is either a necklace or a cycle of length 2. A graph $G$ is extensible between $x$ and $y$ if, $x$ and $y$ being two distinct vertices of $G$, the graph $G_{x y}^{w}$ obtained from $G$ by adding a new vertex $w$ and the two edges $w x$ and $w y$, is minimally 2-edge connected. In particular a graph that is an edge $x y$ is extensible between $x$ and $y$. A graph $G$ is called an $E$-chain if it can be represented by $G_{1} a_{1} G_{2} a_{2} \cdots a_{k-1} G_{k}$ where the following properties are satisfied:

(i) $k \geq 1$.

(ii) $\forall i=1,2, \ldots, k: G_{i}$ is either an edge or a necklace.

(iii) $\forall i=1,2, \ldots, k-1: V\left(G_{i}\right) \cap V\left(G_{i+1}\right)=a_{i}$.

(iv) $\forall i=1,2, \ldots, k-2 \forall j=i+2, \ldots, k: V\left(G_{i}\right) \cap V\left(G_{j}\right)=\emptyset$.

A $G_{i}$ is called a component of the $E$-chain. If $G$ is an $E$-chain, it has a unique decomposition satisfying the preceding conditions. Figure 3.9 shows an example of E-chain graph.

Theorem 3.24 (G. Chaty and M. Chein $[\mathrm{CC}], 1979)$ The family $F_{0}$ of necklace is obtained as follows: 
(1) Every cycle of length $\geq 3$ belongs to $F_{0}$.

(2) If $G_{1}, G_{2}, \ldots, G_{k}, k \geq 2$, is a set of vertex-disjoint graphs such that

(a) for each $i=1, \ldots, k, G_{i}$ belongs to $F_{0}$ or is an edge, and

(b) at least two of them are edges,

then the graph $G$ obtained as follows belongs to $F_{0}$ : one identifies $y_{k}$ and $x_{1}, y_{i}$, and $x_{i+1}$ for each $i=1,2, \ldots, k-1$, where $x_{i}, y_{i} \in V\left(G_{i}\right)$ and $G_{i}$ is extensible between $x_{i}$ and $y_{i}$, for each $i=1,2, \ldots, k$.

Theorem 3.25 (G. Chaty and M. Chein [CC], 1979) The family $F$ of the minimally $(2,2)$-edge connected graphs is obtained as follows:

(1) Each 2-cycle belong to $F$.

(2) $F_{0} \subset F$.

(3) If $G_{1}$ and $G_{2}$ belong to $F$ with $V\left(G_{1}\right) \cap V\left(G_{2}\right)=\emptyset$, then the graph obtained by identifying any vertex of $G_{1}$ with any vertex of $G_{2}$ belongs to $F$. 


\section{Chapter 4}

\section{The lower bound of generalized edge-connectivity and optimal graphs}

We first obtain the lower bound of the generalized $\lambda$. Then, we investigate family of graphs which attains the lower bound. In Sections 4.2 we shall focus on the graph optimization problems arising in network design. A general problem may be stated as follows:

Problem 4.1: Find a graph $H$ satisfying a set of properties $P$ and having an extremal number of edges.

\subsection{Lower bound of $\lambda_{l}$}

We give a lower bound for $\lambda_{l}$ interms of $\lambda_{2}$ and construct families of graphs statisfing the new lower bound with minimum number of edges and with maximum number of 
edges.

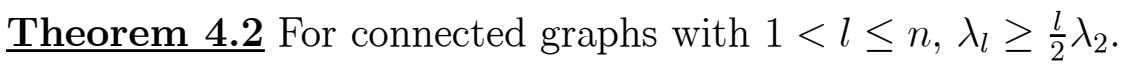

Proof: Let $G$ be a connected graph and $Y$ be a set of $\lambda_{l}(G)$ edges of $G$, such that $G-Y$ has $l$ components $C_{1}, C_{2}, \ldots C_{l}$ of $G-Y$. By the definition of connectivity we have,

$$
\left|\left[V\left(C_{i}\right), V\left(G-C_{i}\right)\right]\right| \geq \lambda(G) \text { for each } i \text { with } 1 \leq i \leq l \text {. }
$$

By taking the sum from $i=1$ to $l$, we obtain,

$$
\sum_{i=1}^{l}\left|\left[V\left(C_{i}\right), V\left(G-C_{i}\right)\right]\right| \geq l \lambda(G) .
$$

Hence

$$
\lambda_{l}(G)=\frac{\sum_{i=1}^{l}\left|\left[V\left(C_{i}\right), V\left(G-C_{i}\right)\right]\right|}{2} \geq \frac{l}{2} \lambda(G)
$$

As an example consider the special case $l=3$ and the graph which has $n$ vertices of degree two, i.e., $n$-cycle $C_{n}$. Clearly, $\lambda_{2}=2$; thus by Theorem $4.2, \lambda_{3} \geq 3$. Notice that this is somewhat better than observing that; $\lambda_{3} \geq 2$ because the $C_{n}$ is connected.

Note that for $l=n, \lambda_{n}=m$. Thus for $l=n$, Theorem 4.2 gives us immediately Theorem 3.1. Another application of Theorem 4.2 would be to find sufficient condition for $\lambda_{l}$ to be greater than or equal to $\delta$.

Corollary 4.3 Let $G$ be a connected graphs with $2 \leq l \leq n$. If $\lambda_{2}(G) \geq\left\lceil\frac{2 \delta}{l}\right\rceil$ then $\lambda_{l} \geq \delta$

\section{Comparison of Theorem 3.8 and Theorem 4.2}

By comparing Theorem 3.8 and Theorem 4.2 one can see that Theorem 3.8 coincides with Theorem 4.2 when $l=3$ and that Theorem 4.2 gives a better bound when 
$l>3$. When $i=l-2$, Theorem 3.8 gives for any connected graphs with $2<l \leq n$, $\lambda_{l} \geq \frac{l-1}{l+1} 3 \lambda_{2}$. Now we compare the coefficients of $\lambda_{2}$. Simple algebraic manipulation yields

$$
\frac{l}{2}>\frac{3(l-1)}{l+1} \Longleftrightarrow(l-2)(l-3)>0 .
$$

Since $l>2$ both theorems are the same for $l=3$ but for $l>3$ the Theorem 4.2 gives a better bound.

In the rest of this chapter we investigate graphs with the following equality,

$$
\lambda_{l}=\frac{l}{2} \lambda
$$

Note that $\lambda_{l}$ is an integer. Thus if (4.1) holds for a graph, then $l \lambda$ must be even.

Lemma 4.4 Let $G$ be a graph satisfying (4.1). Let $Y$ be a set of $\lambda_{l}(G)$ edges of $G$ such that $G-Y$ has $l$ components $C_{1}, C_{2}, \ldots, C_{l}$. Then

$$
\left|\left[V\left(C_{i}\right), V\left(G-C_{i}\right)\right]\right|=\lambda(G) \text { for all } 1 \leq i \leq l .
$$

Proof: By the definition of $\lambda(G)$,

$$
\left|\left[V\left(C_{i}\right), V\left(G-C_{i}\right)\right]\right| \geq \lambda(G) \text { for all } 1 \leq i \leq l .
$$

By (4.1) and by the definition of $Y$,

$$
\frac{1}{2} \sum_{i=1}^{l}\left|\left[V\left(C_{i}\right), V\left(G-C_{i}\right)\right]\right|=|Y|=\lambda_{l}(G)=\frac{l}{2} \lambda(G)
$$

and so we have,

$$
\sum_{i=1}^{l}\left|\left[V\left(C_{i}\right), V\left(G-C_{i}\right)\right]\right|=l \lambda(G) .
$$

It follows by (4.3) and (4.4) that,

$$
\left|\left[V\left(C_{i}\right), V\left(G-C_{i}\right)\right]\right|=\lambda(G) .
$$


Lemma 4.5 Let $G$ be a graph satisfying (4.1). Let $Y$ be a set of $\lambda_{l}(G)$ edges of $G$ such that $G-Y$ has $l$ components $C_{1}, C_{2}, \ldots, C_{l}$. If the component $C_{i}$ has more than one vertex then the number of vertices in $C_{i}$ is at least $\lambda(G)$, for some $i$ with $1 \leq i \leq l$.

Proof: Fix an $i$ with $1 \leq i \leq l$ and let $n_{i}=\left|V\left(C_{i}\right)\right|$. By Lemma 4.4,

$$
\left|\left[V\left(C_{i}\right), V\left(G-C_{i}\right)\right]\right|=\lambda(G) .
$$

Thus $n_{i}\left(n_{i}-1\right)+\lambda(G) \geq$ number of incidences with vertices in $V\left(C_{i}\right) \geq n_{i} \lambda(G)$, and so $\left(n_{i}-\lambda(G)\right)\left(n_{i}-1\right)>0$. Lemma 4.5 now follows by $n_{i}>1$.

Theorem 4.6 Assume that $l \geq 3$ is an integer. Let $G$ be a simple graph with $\lambda(G)=s$ and $\lambda_{l}(G)=t$. Then $G$ satisfies

$$
l s=2 t
$$

if and only if each of the following holds:

(i) $G$ can be contracted to an $s$-regular graph $G^{\prime}$ with $\left|V\left(G^{\prime}\right)\right|=l$ and $\left|E\left(G^{\prime}\right)\right|=t$;

(ii) the preimage of each nontrivial vertex in $G^{\prime}$ has at least $s$ vertices; and

(iii) there is at most one edge joining two trivial vertices in $G^{\prime}$.

Proof: Suppose first (4.5). Then $G$ has $Y \subseteq E(G)$ such that $G-Y$ has $l$ components $C_{1}, C_{2}, \ldots, C_{l}$. Let $X=\cup_{i=1}^{l} E\left(C_{i}\right)$ and $G^{\prime}=G / X$. Then the $l$ components of $G-Y$ are vertices of $G^{\prime}$ and the edges in $Y$ are the edges of $G^{\prime}$. By Lemma 4.5, $\left|\left[V\left(C_{i}\right), V\left(G-C_{i}\right)\right]\right|=\lambda(G)=s$ for all $1 \leq i \leq l$ and so $G^{\prime}$ is an $s$-regular graph. Note that $\left|V\left(G^{\prime}\right)\right|=l$ and $\left|E\left(G^{\prime}\right)\right|=s|V(G)| / 2=s l / 2=t$. This proves (i). Theorem 4.6 (ii) and (iii) follows by Lemma 4.5 and the simpleness of $G$ respectively. 
Conversely, by (i) $G^{\prime}$ is a $s$-regular graph with $\left|V\left(G^{\prime}\right)\right|=l$ and $\left|E\left(G^{\prime}\right)\right|=t$. It is well known that for a $s$-regular graph $G^{\prime},\left|E\left(G^{\prime}\right)\right|=s\left|V\left(G^{\prime}\right)\right| / 2$. Thus $l s=2 t$.

Corollary 4.7 Let $G$ satisfy (4.1) and $G^{\prime}$ be the graph defined in Theorem 4.6. Let $b$ denote the number of nontrivial vertices in $G^{\prime}$. Then

$$
|V(G)| \geq(l-b)+b \lambda .
$$

Now we extend the definition of circulant graphs to define circulant component graphs. Let $G$ be a graph and $T$ be a set of edges of $G$ such that $G-T$ has $l$ components, $C_{0}, C_{1}, \ldots, C_{l-1}$. If a component has only one vertex then it is called a trivial component. Two components $C_{i}$ and $C_{j}$ are said to be adjacent in $G$ if there is a vertex $x$ in $C_{i}$ and vertex $y$ in $C_{j}$ such that the edge $x y \in$ $T$. Let $b_{i}$ be the number of edges between two components. If $b_{i}>1$ then at most only one adjacent component can be trivial. The circulant component graph $C C_{l}\left[a_{1}\left(b_{1}\right), a_{2}\left(b_{2}\right), \ldots, a_{k}\left(b_{k}\right)\right]$ or briefly $C C_{l}\left[a_{i}\left(b_{i}\right)\right]$, where $0<a_{1}<a_{2}<\cdots<a_{k}<$ $\left\lceil\frac{l}{2}\right\rceil$, has $C_{i \pm a_{1}(\bmod l)}, C_{i \pm a_{2}(\bmod l)}, \ldots C_{i \pm a_{k}(\bmod l)}$ adjacent to each component $C_{i}$, with $b_{1}, b_{2}, \ldots, b_{k}$ edges consecutively. Figure 4.1 gives $C C_{5}[1(2), 2(1)]$. Note that the definition of circulant component graph does not say any thing about the structure of the components of $G-T$. In section 4.2 we use circulant component graphs in the construction of minimal. Then all we have to do is to give the structure of each component of $G-T$.

Theorem 4.8 Let $G$ be a circulant component graph and $T$ be an edge-cut of $G$ such that $G-T$ has $l$ components. Then $G$ can be contracted to an $2|T| / l$-regular graph $G^{\prime}$ with $\left|V\left(G^{\prime}\right)\right|=l$ and $\left|E\left(G^{\prime}\right)\right|=|T|$.

Proof: Let $E(G)-T=X$ and $G^{\prime}=G / X$. Then the $l$ components of $G-T$ are vertices 
of $G^{\prime}$ and the edges in $T$ are the edges of $G^{\prime}$. Thus $\left|V\left(G^{\prime}\right)\right|=l$ and $\left|E\left(G^{\prime}\right)\right|=|T|$.

Let $G$ be a circulant component graph and $T$ be an edge-cut of $G$ such that $G-T$ has $l$ components. Let $C$ be a component of $G-T$. A vertex $v$ of $C$ is internal if $v$ is not incident with any edge of $T$; otherwise, $v$ will be external. If $e \in T$ then the edge $e$ joins two external vertices of two different components of $G-T$. Furthermore $e$ is called an external edge of the circulant component graph. Thus all the edges of $T$ are external edges of the circulant component graph. Therefore the definition of the circulant component graph gives only the arrangement of the external edges.

\subsection{Graphs reaching the lower bound with mini- mum number of edges}

Theorem 4.9 Let $G$ be a graph satisfying (4.1), then

$$
\min |E(G)|=\frac{\lambda(G)|V(G)|}{2} .
$$

Proof: In order to prove the theorem we will prove the following two statements:

(i) $|E(G)| \geq \frac{1}{2} \lambda(G)|V(G)|$.

(ii) There exists a graph $H$ satisfying (4.1) such that $|E(H)|=\left\lceil\frac{1}{2} \lambda(H)|V(H)|\right\rceil$.

Proof of (i): Let $T$ be a set of $\lambda_{l}(G)$ edges of $G$ such that $G-T$ has $l$ components $C_{1}, C_{2}, \ldots, C_{l}$. Consider a component $C_{i}$ of $G-T$. Let $v \in V\left(C_{i}\right)$. Then

$$
\operatorname{deg}_{G} v \geq \lambda(G)
$$

Let $\left|V\left(C_{i}\right)\right|=n_{i}$. By Lemma $4.6\left|\left[V\left(C_{i}\right), V\left(G-C_{i}\right)\right]\right|=\lambda(G)$. By (4.8) and by 
Lemma $4.7 \lambda n_{i} \leq \sum_{v \in V\left(C_{i}\right)} \operatorname{deg} v=2\left|E\left(C_{i}\right)\right|+\lambda$ and so $\lambda\left(n_{i}-1\right) \leq 2\left|E\left(C_{i}\right)\right|$. It follows that

$$
\left|E\left(C_{i}\right)\right| \geq \frac{\lambda(G)}{2}\left(n_{i}-1\right) .
$$

Note that $\left|E\left(C_{i}\right)\right|$ is an integer. Thus if the equality of (4.9) holds for a graph $G$, then $\lambda(G)\left(n_{i}-1\right)$ must be even. Thus,

$$
\begin{aligned}
|E(G)| & =\sum_{i=1}^{l}\left|E\left(C_{i}\right)\right|+\lambda_{l}(G) \geq \sum_{i=1}^{l} \frac{\lambda(G)\left(n_{i}-1\right)}{2}+\frac{l}{2} \lambda(G) \\
& =\frac{\lambda(G)}{2}\left(\sum_{i=1}^{l} n_{i}-\sum_{i=1}^{l} 1\right)+\frac{l}{2} \lambda(G)=\frac{\lambda(G)}{2}|V(G)|-\frac{\lambda(G)}{2} l+\frac{l}{2} \lambda(G) \\
& =\frac{\lambda(G)|V(G)|}{2} .
\end{aligned}
$$

Proof of (ii): We shall construct a family of graphs satisfying (4.1) and $|E(H)|=$ $\left\lceil\frac{1}{2} \lambda(H)|V(H)|\right\rceil$. We use terminology from Theorem 4.6 in the construction of graph $H$. Thus $H$ can be contracted to a $s$-regular graph $H^{\prime}$ with $\left|V\left(H^{\prime}\right)\right|=l$ and $\left|E\left(H^{\prime}\right)\right|=t$. We shall prove that such constructed $H$ satisfies $\lambda_{2}(H)=s$ and $\lambda_{l}(H)=t$. It is convenient to give construction separately for even and odd values of $s$.

For even $s$ let $H$ be the graph $C C_{l}[1(s / 2)]$. Figure 4.2 gives the graph $C C_{5}[1(3)]=$ $C C_{5}[1(6 / 2)]$, that is $s=6$ and $l=5$. When $s$ is odd $l$ must be even. Thus let $H$ be the graph $C C_{l}\left[1\left(\frac{s-1}{2}\right), \frac{l}{2}(1)\right]$. Figure 4.3 gives the graph $C C_{6}[1(2), 3(1)]=$ $C C_{6}\left[1\left(\frac{5-1}{2}\right), \frac{6}{2}(1)\right]$, that is $s=5$ and $l=6$. In both Figure 4.2 and Figure 4.3 the structure of the components were not given. Below we define the structure of each component. The edges joining two components are in $T$, thus $H-T$ gives $l$ components $C_{0}, C_{1}, \ldots, C_{l-1}$. Let $C$ be a component of $H-T$. A vertex $v$ of $C$ is internal if $v$ is not incident with any edge of $T$; otherwise, $v$ will be external. Let $C_{i}$ and $C_{j}$ be two distinct components of $H-T$, for $0 \leq i \neq j \leq l-1$. If there is an edge joining a vertex $v$ of $C_{i}$ and a vertex $u$ of $C_{j}$ then this edge $e$ must be in $T$. Further more $C_{i}$ and 
$C_{j}$ are called adjacent components. The edge $e$ is called an external edge of $C_{i}$ and $C_{j}$.

Note that $T$ is an edge subset of $E(H)$, such that $H-T$ has $l$ components. Let $X=E(H)-T$, and $H^{\prime}=H / X$. Thus the vertices of $H^{\prime}$ are components of $H-T$. Also note that the elements of $T$ are edges of $H^{\prime}$. Label the $l$ components of $H-T$ by $C_{0}, C_{1}, \ldots, C_{l-1}$. Now we look at the structure of these components. By Lemma 4.5 , if a component $C_{i}$ has more than one vertex then the number of vertices in $C_{i}$ is at least $s$. Recall that we want to construct graphs with edge-connectivity equals to $s$. Instead of constructing $l$ components $C_{0}, C_{1}, \ldots, C_{l-1}$, we just construct one such component (say $C$ ) and give several different cases. The components of $H-T$ can be any combination of these components provided the components $C_{i}$ and $C_{i+1}$ both cannot be trivial components at the same time for $0 \leq i \leq l$, where component $C_{l}=C_{0}$. Let $|V(C)|=n^{\prime}$. By Lemma 4.5, if $n^{\prime}>1$ then $n^{\prime} \geq s$. Thus we break the construction of $C$ into five cases depending on the values of $n^{\prime}$ and $s$. They are $n^{\prime}=1, n^{\prime}=s, n^{\prime}>s$ for even $s, n^{\prime}>s$ for odd $s$ and even $n^{\prime}$, and $n^{\prime}>s$ for odd $s$ and odd $n^{\prime}$. For all the cases and sub cases label the $n^{\prime}$ vertices $v_{1}, v_{2}, \ldots, v_{s}, v_{s+1}, \ldots, v_{n^{\prime}}$ so that $s$ edges of $T$ meets $v_{1}, v_{2}, \ldots, v_{s}$, when $n^{\prime} \geq s$ in $C$. Thus these $s$ vertices are the external vertices of $C$. All the other vertices are internal.

Case (1): If $n^{\prime}=1$ then the component $C$ is a single vertex. Thus $s$ edges of $T$ meet this vertex. This is a trivial component of $H-T$.

Case (2): If $n^{\prime}=s$ then let the component $C$ be the complete graph $K_{s}$. Note that all the $s$ vertices are incident with $s$ edges of $T$. Thus all the $s$ vertices are external with $\operatorname{deg}_{H} v_{i}=s$ for $1 \leq i \leq s$. 
Case (3): If $n^{\prime}>s$ and $s$ is even. Then let the component $C$ be

$$
C_{n^{\prime}}\left[1,2, \ldots, \frac{s}{2}\right]-\left\{v_{1} v_{2}, v_{3} v_{4}, \ldots, v_{s-1} v_{s}\right\} .
$$

There are $s$ external vertices and $n^{\prime}-s$ internal vertices. $\operatorname{deg}_{H} v_{i}=s$ for $1 \leq i \leq n^{\prime}$. The graph $C_{7}[1,2,3]-\left\{v_{1} v_{2}, v_{3} v_{4}, v_{5} v_{6}\right\}$ is shown in the Figure 4.4.

Case (4): If $n^{\prime}>s, s$ is odd and $n^{\prime}$ is even. Then let the component $C$ be

$$
C_{n^{\prime}}\left[1,2, \ldots, \frac{s-1}{2}, \frac{n^{\prime}}{2}\right]-\left\{v_{2} v_{3}, v_{4} v_{5}, \ldots, v_{s-1} v_{s}\right\} .
$$

There are $s$ external vertices and $n^{\prime}-s$ internal vertices. $\operatorname{deg}_{H} v_{1}=s+1$ and $\operatorname{deg}_{H} v_{i}=s$ for all $2 \leq i \leq n^{\prime}$. The graph $C_{6}[1,2,3]-\left\{v_{2} v_{3}, v_{4} v_{5}\right\}$ is shown in the Figure 4.5.

Case (5) If $n^{\prime}>s$ and $s$ and $n^{\prime}$ are both odd. This we break into two sub cases as $n^{\prime}<2 s$ and $n^{\prime}>2 s$.

Subcase (5a): $s<n^{\prime}<2 s$ for odd $s$ and $n^{\prime}$. Then let the component $C$ be $C_{n^{\prime}}\left[1,2, \ldots, \frac{s-1}{2}\right]+\left\{v_{s+1} v_{t+1}, v_{s+2} v_{t+2}, \ldots, v_{n^{\prime}} v_{t+n^{\prime}-s}\right\}-\left\{v_{t+1} v_{t+2}, v_{t+3} v_{t+4}, \ldots, v_{t+n^{\prime}-s-1} v_{t+n^{\prime}-s}\right\}$.

Where $t=\left(\frac{3 s+1}{2}\right) \bmod n^{\prime}$. There are $s$ external vertices and $n^{\prime}-s$ internal vertices. $\operatorname{deg}_{H} v_{i}=s$ for $1 \leq i \leq n^{\prime}$. The graph $C_{7}[1,2]+\left\{v_{2} v_{6}, v_{3} v_{7}\right\}-\left\{v_{2} v_{3}\right\}$ is shown in Figure 4.6.

Subcase (5b): $n^{\prime}>2 s$ for odd $s$ and $n^{\prime}$. Then let the component $C$ be

$$
C_{n^{\prime}}\left[1,2, \ldots, \frac{s-1}{2}\right]+\left\{v_{t+s+1} v_{s+1}, v_{t+s+2} v_{s+2}, \ldots, v_{n^{\prime}} v_{t+s}\right\} .
$$

Where $t=\frac{n^{\prime}-s}{2}$. There are $s$ external vertices and $n^{\prime}-s$ internal vertices. $\operatorname{deg}_{H} v_{i}=s$ for $1 \leq i \leq n^{\prime}$. The graph $C_{11}[1,2]+\left\{v_{6} v_{9}, v_{7} v_{10}, v_{8} v_{11}\right\}$ is shown in Figure 4.7. 
We want to find the edge connectivity of the component $C$ in $H$. The component $C$ has $s$ external edges. To find the edge-connectivity of $C$ with these $s$ external edges assume the edges incident with a vertex $v$ of $H-C$. Recall that in the graph $H$ the $s$ external edges never incident with a one vertex. Thus the above assumption is made only to find the edge-connectivity of the component $C$. This assumption does not change the edge-connectivity of $C$ in $H$. Denote this graph by $C \cup v$. We would like to prove $\lambda(C \cup v)=s$ for each case raised in the construction of the component $C$.

Lemma 4.10 For any component $\mathrm{C}$ in the above construction $\lambda(C \cup v)=s$.

Proof Let $X$ be an edge cut of $C \cup v$ such that $\lambda(C \cup v)=|X|$. If the edge cut $X$ separate the vertex $v$ and the component $C$ then $|X|=s$. In following cases we assume that the edge-cut $X$ does not separate the vertex $v$ and the component $C$.

Case (1): $n^{\prime}=1$. Thus the graph $C \cup v$ has only two vertices and $s$ edges between them. Thus the edge-cut $X$ separates the vertex $v$ and the component $C$. Therefore $|X|=s$ and $\lambda(C \cup v)=s$.

Case (2): $n^{\prime}=s$. Thus the graph $\lambda(C \cup v)$ is the complete graph $K_{s+1}$. Therefore $|X|=s$ and $\lambda(C \cup v)=s$.

Case (3): $n^{\prime}>s$ for even $s$. Recall that for this case the component $C=C_{n^{\prime}}\left[1,2, \ldots, \frac{s}{2}\right]-$ $\left\{v_{1} v_{2}, v_{3} v_{4}, \ldots, v_{s-1} v_{s}\right\}$. In the construction of $C$ first we construct $C_{n^{\prime}}\left[1,2, \ldots, \frac{s}{2}\right]$. The edge-connectivity $\lambda\left(C_{n^{\prime}}\left[1,2, \ldots, \frac{s}{2}\right]\right)=s$. After removing the edges $\left\{v_{1} v_{2}, v_{3} v_{4}, \ldots, v_{s-1} v_{s}\right\}$ the edge-connectivity is reduced by one. Therefore $\lambda\left(C_{n^{\prime}}\left[1,2, \ldots, \frac{s}{2}\right]-\left\{v_{1} v_{2}, v_{3} v_{4}, \ldots, v_{s-1} v_{s}\right\}\right)=$ $s-1$. That is $\lambda(C)=s-1$. When we connect $s$ external vertices to the vertex $v$ the 
edge-connectivity is increased by one. Thus $\lambda(C \cup v)=s$.

Case (4): $n^{\prime}>s$ for odd $s$ and even $n^{\prime}$. In this case the component $C=C_{n^{\prime}}\left[1,2, \ldots, \frac{s-1}{2}, \frac{n^{\prime}}{2}\right]-$ $\left\{v_{2} v_{3}, v_{4} v_{5}, \ldots, v_{s-1} v_{s}\right\}$. The edge-connectivity $\lambda\left(C_{n^{\prime}}\left[1,2, \ldots, \frac{s-1}{2}, \frac{n^{\prime}}{2}\right]\right)=s$. Therefore $\lambda(C)=s-1$. Thus $\lambda(C \cup v)=s$.

Subcase (5a): $s<n^{\prime}<2 s$ for odd $s$ and $n^{\prime}$. In this case the component $C=$ $C_{n^{\prime}}\left[1,2, \ldots, \frac{s-1}{2}\right]+\left\{v_{t+s+1} v_{s+1}, v_{t+s+2} v_{s+2}, \ldots, v_{n^{\prime}} v_{t+s}\right\}$. The edge-connectivity $\lambda\left(C_{n^{\prime}}\left[1,2, \ldots, \frac{s-1}{2}\right]\right)=$ $s$. Therefore $\lambda(C)=s-1$. Thus $\lambda(C \cup v)=s$.

$\underline{\text { Subcase (5b): }} n^{\prime}>2 s$ for odd $s$ and $n^{\prime}$. In this case the component $C=C_{n^{\prime}}\left[1,2, \ldots, \frac{s-1}{2}\right]+$ $\left\{v_{t+s+1} v_{s+1}, v_{t+s+2} v_{s+2}, \ldots, v_{n^{\prime}} v_{t+s}\right\}$. Where $t=\frac{n^{\prime}-s}{2}$. The edge-connectivity $\lambda\left(C_{n^{\prime}}\left[1,2, \ldots, \frac{s-1}{2}\right]\right)=$ $s$. Therefore $\lambda(C)=s-1$. Thus $\lambda(C \cup v)=s$.

Now we prove the following two statements:

(I) $\lambda_{2}(H)=s$.

(II) $\lambda_{l}(H)=t$.

Proof of (I): If we remove a set of edges incident a component $C_{i}$ for any $1 \leq i \leq l$ then we get two components. In the construction every component incident with exactly $s$ edges. Thus $\lambda_{2}(H) \leq s$. By contradiction assume that there is an edge-cut $E^{\prime} \subseteq E(H)$ such that $H-E^{\prime}$ has two components and $\left|E^{\prime}\right|<s$. If $E^{\prime} \subset T$ then $E^{\prime}$ separates a component $C_{i}$. Thus $\left|E^{\prime}\right|=s$. If $E^{\prime} \subset E(H)-T$ then by Lemma 4.10 $\left|E^{\prime}\right| \geq s$. Therefore $\left|E^{\prime}\right| \geq s$. Thus $\lambda_{2}(H)=s$.

Proof of (II): By Theorem 4.7 and by $\lambda_{2}(H)=s$, the $l$-edge-connectivity of $H$ is at 
least $\left\lceil\frac{l s}{2}\right\rceil$. Therefore it suffices to show that $H$ has an edge subset $Y$ such that $H-Y$ has at least $l$ components and such that $|T|=\left\lceil\frac{l s}{2}\right\rceil$ If we remove all the $T$ edges of $G$, then the resulting graph has $l$ components and $|T|=\left\lceil\frac{l s}{2}\right\rceil=t$. Thus $\lambda_{l}(H)=t$. This complete the proof of Theorem 4.9 . 


\section{Chapter 5}

\section{Relationship between generalized edge-connectivity and \\ edge-strength}

The main results of this Chapter has been accepted for publication [HLX]. We will find an upper bound for generalized edge-connectivity, in this chapter. First we state a general upper bound. We know $\lambda_{l} \leq m$. For $2 \leq l \leq n$, the following property states a better upper bound than $\lambda_{l} \leq m$.

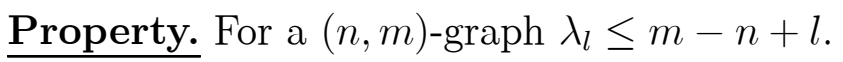

Proof. Let $G$ be an $(n, m)$-graph. Then $\lambda_{n}(G)=m$. That is by removing all the edges we get $n$ components. All the components are $K_{1}$, single vertex. If we put one edge back into the graph that edge will join two vertices and will give $n-1$ components. Thus $\lambda_{n-1}(G)=m-1$. We keep doing this till we get $l$ components. Since $\lambda_{l}$ being the smallest number of edges to get $l$ components gives the inequality 
$\lambda_{n-m^{\prime}}(G) \leq m-m^{\prime}$, after adding $m^{\prime}$ edges back in to the graph. Thus for $l$ components, set $l=n-m^{\prime}$, which in turn gives the inequality $\lambda_{l}(G) \leq m-n+l$.

Let $G$ be an $(n, m)$-graph and $C$ be a cycle of $G$. When all the edges were removed from $G$, it gives $n$ components. Now we put one edge back at a time. At one point it would complete the cycle $C$ and wouldn't change the number of components. Thus if $G$ contains a cycle then $\lambda_{l}(G)<m-n+1$, for some $2 \leq l \leq n$. Therefore graphs attain the upper bound $\lambda_{l} \leq m-n+l$ only when it doesn't have a cycle. Thus only trees attain the upper bound $\lambda_{l}(G)=m-n+l$. Therefore we need to find a better upper bound for $\lambda_{l}$. We will prove that $\lambda_{l} \leq(l-1) \bar{\lambda}(G)$, and characterize the graphs when equality holds for $\lambda(G)=2$ and for all values of $l \geq 2$. As a byproduct, we characterize graphs with $\lambda(G)=\bar{\lambda}(G)=2$. To prove these results we need the concept of contraction. Therefor as we go along we will prove some properties of contraction.

\section{$5.1 \quad l$-sequential cuts}

Let $G$ be a graph with $|V(G)| \geq 2$. An $l$-sequential cut is a sequence $\left\{X_{1}, X_{2}, \cdots, X_{l-1}\right\}$

of edge subsets of $G$ such that $X_{i}$ is an edge-cut of a component of $G_{i}=G-\cup_{j=0}^{i} X_{j}$ such that $k\left(G_{i}\right)+1=k\left(G_{i}-X_{i+1}\right)$, where $X_{0}=\emptyset$. The sequence $\left\{X_{1}, X_{2}, \cdots, X_{l-1}\right\}$ is a minimal $l$-sequential cut if each $X_{i}$ is a minimum edge-cut of $G_{i}$. The sequence $\left\{X_{1}, X_{2}, \cdots, X_{l-1}\right\}$ is a minimum $l$-sequential cut if, among all $l$-sequential cuts of $G, \sum_{i=1}^{l-1}\left|X_{i}\right|$ is minimum.

Lemma 5.1 Let $G$ be a graph with $|V(G)| \geq 2$. $G$ has an $l$-sequential cut if and only if $|V(G)| \geq l$. 
Proof Let the sequence $\left\{X_{1}, X_{2}, \cdots, X_{l-1}\right\}$ be an $l$-sequential cut. Then $G-\left\{X_{1}, X_{2}, \cdots, X_{l-1}\right\}$ has $l$ non empty components $C_{1}, C_{2}, \cdots, C_{l}$, and so $|V(G)|=\sum_{i=1}^{l}\left|V\left(C_{i}\right)\right| \geq l$.

Conversely, we shall prove that if $|V(G)| \geq l$ then $G$ has an $l$-sequential cut by induction on $l$. For the case $l=2,|V(G)| \geq 2$. Let $u \in V(G)$, such that $u$ is not a cut vertex. Let $X_{1}$ be the set of edges incident to $u$. Then since $u$ is not a cut vertex, $X_{1}$ is a bond of $G$. Thus $G-X_{1}$ separate $G$ into two components $u$ and $G-u$. Thus $X_{1}$ is a 2-sequential cut, and the result holds for $l=2$. Assume the result hold for $l=s-1$. That is if $|V(G)| \geq s-1$ then $G$ has an $(s-1)$-sequential cut. Now if $|V(G)| \geq s$, by induction $G$ has an $(s-1)$-sequential cut $\left\{X_{1}, X_{2}, \cdots, X_{s-2}\right\}$ such that $G-\left\{X_{1}, X_{2}, \cdots, X_{s-2}\right\}$ has $(s-1)$ components $C_{1}, C_{2}, \cdots, C_{s-1}$. Since $|V(G)| \geq s$ at least one component $C_{i}$ for $1 \leq i \leq s-1$ has more than one vertex. Thus if $\left|V\left(C_{i}\right)\right| \geq 2$ we can find a bond $X_{s-1}$ such that $C_{i}$ is separated into 2-components. Therefore $\left\{X_{1}, X_{2}, \cdots, X_{s-1}\right\}$ is an $l$-sequential cut.

Lemma 5.2 If $\left\{X_{1}, \cdots, X_{l-1}\right\}$ is a minimum $l$-sequential cut of $G$, then there is a minimum $l$-sequential cut $\left\{X_{1}^{\prime}, \cdots, X_{l-1}^{\prime}\right\}$ such that for each $i$ with $1 \leq i \leq l-1, X_{i}^{\prime}$ is a bond of a component of $G_{i}=G-\cup_{j=0}^{i} X_{j}$, where $X_{0}=\emptyset$.

Proof Let $\left\{X_{1}, \cdots, X_{l-1}\right\}$ be a minimum $l$-sequential cut of $G$. Define $\left\{X_{1}^{\prime}, \cdots, X_{l-1}^{\prime}\right\}$ as follows.

Let $X_{1}^{\prime} \subseteq X_{1}$ be a minimal edge-cut of $G$, and after $X_{1}^{\prime}, X_{2}^{\prime}, \cdots, X_{m}^{\prime}$ are defined, for some $m$ with $1 \leq m<l-1$, let $X_{m+1}^{\prime} \subseteq \cup_{i=1}^{m+1} X_{i}-\cup_{i=1}^{m} X_{i}^{\prime}$ be a minimal edge-cut of $G-\cup_{i=1}^{m} X_{i}^{\prime}$. By the way $\left\{X_{1}^{\prime}, \cdots, X_{l-1}^{\prime}\right\}$ is defined, it is an $l$-sequential cut of $G$.

Note that the $X_{1}^{\prime}, \cdots X_{l-1}^{\prime}$ are disjoint and that $\cup_{i=1}^{l-1} X_{i}^{\prime} \subseteq \cup_{i=1}^{l-1} X_{i}$, and so $\sum_{i=1}^{l-1}\left|X_{i}^{\prime}\right| \leq$ $\sum_{i=1}^{l-1}\left|X_{i}\right|$. But equality must hold since $\left\{X_{1}^{\prime}, \cdots, X_{l-1}^{\prime}\right\}$ is an $l$-sequential cut of $G$ 
and since $\left\{X_{1}, \cdots, X_{l-1}\right\}$ is minimum.

Observation 5.3 Let $H \subseteq G$. Then each of the following holds:

(i) $\lambda_{2}(G / E(H)) \geq \lambda_{2}(G)$.

(ii) If $\lambda_{2}(G / E(H)) \geq k$ and $\lambda_{2}(H) \geq k$ then $\lambda_{2}(G) \geq k$.

Proof (i). Let $H$ be a subgraph of $G$. Then $E(G / E(H)) \subseteq E(G)$ and any edge-cut of $G / E(H)$ is also an edge-cut of $G$. Thus $\lambda_{2}(G / E(H))$ edge-cut separates the graph $G$ into at least two components, which may not be the minimum cardinality of an edge-cut of $G$.

(ii). Let $X \subseteq E(G)$ be a minimal edge-cut of $G$, and let $X_{1}=X \cap E(H)$ and $X_{2}=X-X_{1}=X \cap E(G / H)$. If $X_{1} \neq \emptyset$, then since $X$ is an edge-cut of $G, X_{1}$ must be an edge-cut of $H$. It follows that $|X| \geq\left|X_{1}\right| \geq k$, by the assumption that $\lambda_{2}(H) \geq k$. If $X_{1}=\emptyset$, then since $X$ is an edge-cut of $G, X_{2}$ must be an edge-cut of $G / E(H)$. Therefore $|X|=\left|X_{2}\right| \geq k$, by the assumption that $\lambda_{2}(G / E(H)) \geq k$.

Lemma 5.4 Let $H$ be a connected graph with $l$ vertices. The vertices of $H$ can be labeled as $v_{1}, v_{2}, \cdots, v_{l}$ so that for each $i(1 \leq i \leq l)$ the graph $H-\left\{v_{1}, v_{2}, \cdots, v_{i}\right\}$ is connected.

Proof We prove this by induction on $|V(H)|$. If $|V(H)|=1$ there is nothing to prove. Suppose the result is true for $|V(H)|<l$. Let $H$ be a connected graph with $l$ vertices. Let $H_{1}$ be a spanning tree of $H$. Label a vertex of degree 1 in $H_{1}$ by $v_{1}$. Thus $H-v_{1}$ is a connected graph since $H_{1}-v_{1}$ is a spanning tree of $H-v_{1}$. Furthermore $\left|V\left(H-v_{1}\right)\right|<l$. Therefore the vertices of $H-v_{1}$ can be labeled as $v_{2}, v_{3}, \cdots, v_{l}$ so 
that for each $i(2 \leq i \leq l)$ the graph $\left(H-v_{1}\right)-\left\{v_{2}, v_{3}, \cdots, v_{i}\right\}$ is connected. Thus for each $i(1 \leq i \leq l)$ the graph $H-\left\{v_{1}, v_{2}, \cdots, v_{i}\right\}$ is connected.

Lemma 5.5 Let $l \geq 2$ be an integer and let $G$ be a connected graph with $|V(G)| \geq l$. Then the followings are equivalent.

(i) There is an edge subset $T$ of $E(G)$ such that $G-T$ has $l$ components.

(ii) $G$ has an $l$-sequential cut $\left\{X_{1}, X_{2}, \cdots, X_{l-1}\right\}$ such that $T=\cup_{i=1}^{l-1} X_{i}$.

Proof Let $T \subseteq E(G)$ such that $G-T$ has $l$ components. Obtain a graph $H$ from $G$ by contracting each component of $G-T$ into a vertex. Thus $H$ has $l$ vertices and $|T|$ edges. Further more $H$ is connected. By Lemma 5.4, the vertices of $H$ can be labeled as $v_{1}, v_{2}, \cdots, v_{l}$ such that $H-\left\{v_{1}, v_{2}, \cdots, v_{i}\right\}$ is connected for all $i$. Let $C_{1}, C_{2}, \cdots, C_{l}$ be the $l$-components of $G-T$, and the component $C_{i}$ is contracted to the vertex $v_{i}$. Let

$$
X_{1}=\left\{e \in T: e \text { has exactly one end in } C_{1}\right\}
$$

and let

$$
X_{i}=\left\{e \in T-\cup_{j=1}^{i-1} X_{j}: e \text { has exactly one end in } C_{i}\right\} .
$$

Since $H-\left\{v_{1}, v_{2}, \cdots, v_{i}\right\}$ is connected, each $X_{i}$ is a bond of $G-\cup_{j=0}^{i-1} X_{j}$, where $X_{0}=\emptyset$. Thus $\left\{X_{1}, X_{2}, \cdots, X_{l-1}\right\}$ is an $l$-sequential cut of $G$ and $T=\cup_{i=1}^{l-1} X_{i}$.

Conversely assume $G$ has a $l$-sequential cut $\left\{X_{1}, X_{2}, \cdots, X_{l-1}\right\}$ such that $T=$ $\cup_{i=1}^{l-1} X_{i}$. By the definition of an $l$-sequential cut, $G-T$ has $l$ components.

Lemma 5.6 Let $l \geq 2$ be an integer and let $G$ be a graph with $|V(G)| \geq l$. Then the followings are equivalent.

(i) There is an edge subset $T$ of $E(G)$ such that $G-T$ has at least $l$ components 
and $\lambda_{l}(G)=|T|$.

(ii) $G$ has a minimum $l$-sequential cut $\left\{X_{1}, X_{2}, \cdots, X_{l-1}\right\}$ such that $T=\cup_{i=1}^{l-1} X_{i}$.

Proof Let $T$ be an edge subset of $E(G)$ such that $G-T$ has at least $l$ components and $\lambda_{l}(G)=|T|$. Since $\lambda_{l}(G)=|T|, G-T$ has exactly $l$ components. Thus by Lemma $5.5 G$ has an $l$ sequential cut. Let $\left\{X_{1}, X_{2}, \cdots, X_{l-1}\right\}$ be an $l$-sequential cut of $G$. Let $\left\{Y_{1}, Y_{2}, \cdots Y_{l-1}\right\}$ be a minimum $l$-sequential cut of $G$. Then $G-\cup_{i=1}^{l-1} Y_{i}$ has $l$ components, and so

$$
\lambda_{l}(G) \leq \sum_{i=1}^{l-1}\left|Y_{i}\right| \leq \sum_{i=1}^{l-1}\left|X_{i}\right|=|T|=\lambda_{l}(G),
$$

which implies $\left\{X_{1}, X_{2}, \cdots, X_{l-1}\right\}$ is a minimum $l$-sequential cut of $G$.

Conversely assume $G$ has a minimum $l$-sequential cut $\left\{X_{1}, X_{2}, \cdots, X_{l-1}\right\}$ such that $T=\cup_{i=1}^{l-1} X_{i}$. By the definition of $l$-sequential cut, $G-T$ has $l$ components thus $\lambda_{l}(G) \leq|T|$. Let $T_{1}$ be an edge subset of $G$ such that $\lambda_{l}(G)=\left|T_{1}\right|$ and $G-T_{1}$ has exactly $l$ components. By Lemma $5.5 G$ has an $l$-sequential cut $\left\{Y_{1}, Y_{2}, \cdots, Y_{l-1}\right\}$ such that $T_{1}=\cup_{i=1}^{l-1} Y_{i}$. Therefore, $T=\cup_{i=1}^{l-1} X_{i}$ and $\left\{X_{1}, X_{2}, \ldots, X_{l-1}\right\}$ is minimum, $\lambda_{l}(G)=\left|T_{1}\right| \geq|T|=\sum_{i=1}^{l-1}\left|X_{i}\right|$. Thus $\lambda_{l}(G)=|T|$.

Lemma 5.7 For any connected graph $G$ with $|V(G)| \geq l,(l-1) \bar{\lambda}_{2}(G) \geq \lambda_{l}(G)$.

Proof Let $\left\{X_{1}, X_{2}, \cdots, X_{l-1}\right\}$ be a minimal $l$-sequential cut. Then

$$
\lambda_{l}(G) \leq \sum_{i=1}^{l-1}\left|X_{i}\right| \leq \sum_{i=1}^{l-1} \bar{\lambda}_{2}(G)=(l-1) \bar{\lambda}_{2}(G) .
$$

Theorem 5.8 Let $G$ be a simple connected graph. Then $(l-1) \bar{\lambda}_{2}(G)=\lambda_{l}(G)$ if and only if each of the following holds: 
(i) any minimal $l$-sequential cut is a minimum $l$-sequential cut.

(ii) for some integer $k, \bar{\lambda}_{2}(G)=\lambda_{2}(G)=k$.

(iii) for any minimal $l$-sequential cut $\left\{X_{1}, X_{2}, \cdots, X_{l-1}\right\} ;\left|X_{i}\right|=k$.

Proof Assume $(l-1) \bar{\lambda}_{2}(G)=\lambda_{l}(G)$. Let $\left\{X_{1}, X_{2}, \cdots, X_{l-1}\right\}$ be a minimal $l$-sequential cut. Then since each $X_{i}$ is a minimum edge cut of a subgraph of $G,\left|X_{i}\right| \leq \bar{\lambda}_{2}(G)$. It follows that

$$
(l-1) \bar{\lambda}_{2}(G)=\lambda_{l}(G) \leq \sum_{i=1}^{l-1}\left|X_{i}\right| \leq(l-1) \bar{\lambda}_{2}(G)
$$

and so equality must hold. This gives (i), and $\left|X_{1}\right|=\left|X_{2}\right|=\cdots=\left|X_{l-1}\right|=\bar{\lambda}_{2}(G)$. By the definition of a minimal $l$-sequential cut, $\left|X_{1}\right|=\lambda_{2}(G)$. Hence $\lambda_{2}(G)=\bar{\lambda}_{2}(G)$. Denote this common value by $k$. Then both (ii) and (iii) hold.

Conversely, by (iii), if $\left\{X_{1}, X_{2}, \cdots, X_{l-1}\right\}$ is a minimal $l$-sequential cut, then $\sum_{i=1}^{l-1}\left|X_{i}\right|=(l-1) k$. By (ii) $\sum_{i=1}^{l-1}\left|X_{i}\right|=(l-1) \bar{\lambda}_{2}(G)$. By (i) $\left\{X_{1}, X_{2}, \cdots, X_{l-1}\right\}$ is a minimum $l$-sequential cut. Hence by Lemma 5.6, $\lambda_{l}(G)=\sum_{i=1}^{l-1}\left|X_{i}\right|$. Thus $(l-1) \bar{\lambda}_{2}(G)=\lambda_{l}(G)$.

The next theorem indicates that graphs reaching the upper bound $(l-1) \bar{\lambda}_{2}(G)=$ $\lambda_{l}(G)$ possesses an interesting property: nontrivial components of such graphs when a minimum edge-cut is removed inherit the same equality. Such a property shall play an important role in the study of extremal graphs.

Theorem 5.9 Let $G$ be a simple graph with $|V(G)| \geq l \geq 3$ and assume $\lambda_{2}(G)>1$ and $(l-1) \bar{\lambda}_{2}(G)=\lambda_{l}(G)$. Let $X \subseteq E(G)$ be a minimum edge-cut of $G$ such that $G-X$ has two components $H_{1}$ and $H_{2}$. Then either $H_{1}=K_{1}$ or $H_{1}$ satisfies

(i) $\lambda_{2}\left(H_{1}\right)=\lambda_{2}(G)$; and 
(ii) $(l-2) \bar{\lambda}_{2}\left(H_{1}\right)=\lambda_{l-1}\left(H_{1}\right)$.

Proof Assume $H_{1} \neq K_{1}$. If $1<\left|V\left(H_{1}\right)\right|=l_{1}<l-1$, then by Lemma 5.1, $H_{1}$ has a minimal $l_{1}$-sequential cut $\left\{W_{2}, W_{3}, \cdots, W_{l_{1}}\right\}$. Note that $\left|W_{l_{1}}\right|=1$, since $H_{1}$ is a simple graph. Since $|V(G)| \geq l$, the minimal $l_{1}$-sequential cut $\left\{W_{2}, W_{3}, \cdots, W_{l_{1}}\right\}$ of $H_{1}$ together with $X$ becomes an $\left(l_{1}+1\right)$-sequential cut $\left\{X, W_{2}, \cdots, W_{l_{1}}\right\}$ in $G$. Since $X$ is a minimum edge-cut of $G,\left\{X, W_{2}, \cdots, W_{l_{1}}\right\}$ is a minimal $\left(l_{1}+1\right)$-sequential cut of $G$. Since $\left|V\left(H_{2}\right)\right|=|V(G)|-\left|V\left(H_{1}\right)\right| \geq l-l_{1}$ and since $H_{2}$ is connected, it follows from Lemma 5.1 that the minimal $\left(l_{1}+1\right)$-sequential cut $\left\{X, W_{2}, W_{3}, \cdots, W_{l_{1}}\right\}$ may be extended to a minimal $l$-sequential cut $\left\{X, W_{2}, W_{3}, \cdots, W_{l_{1}}, \cdots, W_{l-1}\right\}$. But then, by Theorem 5.8(ii) and Theorem 5.8(iii), one must have, for each $i,\left|W_{i}\right|=\lambda_{2}(G)>1$, contrary to $\left|W_{l_{1}}\right|=1$.

Thus $\left|V\left(H_{1}\right)\right| \geq l-1$. By Lemma $5.1, H_{1}$ has a minimal $(l-1)$-sequential cut $\left\{Y_{2}, Y_{3}, \cdots, Y_{l-1}\right\}$. Then $\left\{X, Y_{2}, \cdots, Y_{l-1}\right\}$ is a minimal $l$-sequential cut of $G$. By definition of minimal sequential cut and by Theorem 5.8(iii), $\lambda_{2}\left(H_{1}\right)=\left|Y_{2}\right|=|X|$. Since $X$ is a minimum cut and by Theorem 5.8(ii) and Theorem 5.8(iii) $|X|=\lambda_{2}(G)=$ $\bar{\lambda}_{2}(G)$. Thus $\lambda_{2}\left(H_{1}\right)=\lambda_{2}(G)$, and so (i) holds.

Let $\left\{Z_{2}, \cdots, Z_{l-1}\right\}$ be a minimum $(l-1)$-sequential cut of $H_{1}$. By Lemma 5.6

$$
\lambda_{l-1}\left(H_{1}\right)=\sum_{i=2}^{l-1}\left|Z_{i}\right| .
$$

Since $\left\{X, Z_{2}, \cdots, Z_{l-1}\right\}$ is an $l$-sequential cut of $G$, and since $\left\{X, Y_{2}, \cdots, Y_{l-1}\right\}$ is a minimum $l$-sequential cut of $G$,

$$
|X|+\lambda_{l-1}\left(H_{1}\right)=|X|+\sum_{i=2}^{l-1}\left|Z_{i}\right| \geq|X|+\sum_{i=2}^{l-1}\left|Y_{i}\right| \geq|X|+\lambda_{l-1}\left(H_{1}\right) .
$$

Therefore, equalities must hold everywhere, and so $\lambda_{l-1}\left(H_{1}\right)=\sum_{i=2}^{l-1}\left|Y_{i}\right|$. By Theorem 5.8, $\left|Y_{i}\right|=\bar{\lambda}_{2}(G)$. Therefore

$$
\lambda_{l-1}\left(H_{1}\right)=\sum_{i=2}^{l-1}\left|Y_{i}\right|=\bar{\lambda}_{2}(G)(l-2)=\lambda_{2}\left(H_{1}\right)(l-2) .
$$


Note that $\lambda_{2}\left(H_{1}\right) \leq \bar{\lambda}_{2}\left(H_{1}\right) \leq \bar{\lambda}_{2}(G)$, and so $\bar{\lambda}_{2}\left(H_{1}\right)(l-2)=\lambda_{l-1}\left(H_{1}\right)$ follows.

\subsection{Characterization of Extremal Graphs}

An elementary subdivision of a nonempty graph $H$ is a graph obtained from $H$ by removing some edge $e=u v$ and by adding a new vertex $w$ and new edges $u w$ and $w v$. A subdivision of $H$ is a graph obtained from $H$ by a succession of elementary subdivisions (including the possibility of none). A subdivision of a graph $H$ is denoted by $S(H)$.

Let $H$ be a graph with a specified distinguished vertex $v$. Let $v_{1}, v_{2}, \cdots, v_{d}$ be the vertices on $H$ adjacent to $v$. Let $L$ be a graph disjoint from $H$. Then $H(L)$ denotes the collection of simple graphs obtained from the disjoint union of $L$ and $H-v$ by adding $d$ new edges $e_{1}, e_{2}, \cdots, e_{d}$ such that $e_{i}$ joins $v_{i}$ to a vertex in $L$, for $1 \leq i \leq d$. If $G \in H(L)$, then we say that $G$ is obtained by replacing a vertex $v$ in $H$ by the graph $L$.

Observation 5.10 If $G \in H(L)$, then $L$ is a subgraph of $G(L \subseteq G)$ and $G / E(L)=$ $H$. Thus $E(G)=E(H) \cup E(L)$.

Proof Let $G \in H(L)$. From the definition of $H(L), L$ is a subgraph of of $G$. We get $G / E(L)=H$, by the definition of contraction and by the definition of $H(L)$. Thus $E(G)=E(H) \cup E(L)$.

Let $M(2)$ be a collection of simple graphs having $K_{1}$ (a single vertex graph without edges), and $C_{3}$ (a cycle of length 3 ), such that $G \in M(2)-\left\{K_{1}, C_{3}\right\}$ if and only if either

(M1) $G=S(H)$ for some graph $H \in M(2)$, or 
(M2) $G$ can be obtained from a graph $H \in M(2)$ by replacing a vertex of $H$ by a graph in $\mathrm{M}(2) ;$ i. e., $G \in H(L)$, for some $L \in M(2)$.

Theorem 5.11 Let $G$ be a simple connected graph. Then $\lambda_{2}(G)=\bar{\lambda}_{2}(G)=2$ if and only if $G \in M(2)-\left\{K_{1}\right\}$.

Proof Suppose $G \in M(2)-\left\{K_{1}\right\}$. Let $C_{n}$ denote the $n$-cycle. Note that $\lambda_{2}\left(C_{n}\right)=2$ and $\bar{\lambda}_{2}\left(C_{n}\right)=2$. Thus for $G=C_{n}$, the result is trivial. For any other graph $G \in M(2)-\left\{K_{1}\right\}$ we shall prove $\lambda_{2}(G)=\bar{\lambda}(G)=2$ by induction on $|V(G)|$.

Note first that if $G=S(H)$ for some $H \in M(2)$, then $\lambda_{2}(G)=\lambda_{2}(H)=\bar{\lambda}_{2}(G)=$ $\bar{\lambda}_{2}(H)=2$. Hence we only need to consider the case when $G \in H(L)$, for some $H, L \in M(2)$. We shall show $\lambda_{2}(G)=\bar{\lambda}_{2}(G)=2$. If $\lambda_{2}(G)=1$, then $G$ has an edge-cut $e$. By Observation 5.10, either $e \in E(H)=E(G / L)$ or $e \in E(L)$. By induction, we have $\lambda_{2}(H)=2, \lambda_{2}(L)=2$, and so $e$ cannot be an edge-cut of $G$. Thus $\lambda_{2}(G) \geq 2$.

If $G$ has a subgraph $\Gamma$ with $\lambda(\Gamma) \geq 3$, then by Observation 5.3 , either $\Gamma / \Gamma \cap L$ is a subgraph of $H=G / L$, whence $\lambda_{2}(\Gamma / \Gamma \cap L) \geq \lambda_{2}(\Gamma)$, contrary to $\bar{\lambda}_{2}(H)=2$; or $\Gamma \subseteq L$, contrary to $\bar{\lambda}_{2}(L)=2$. Therefore $\bar{\lambda}_{2}(G)=2$.

Conversely, assume that $\lambda_{2}(G)=\bar{\lambda}_{2}(G)=2$. We argue by induction on $|V(G)|$ to show that $G \in M(2)$. Since $\lambda_{2}(G)=\bar{\lambda}_{2}(G)=2$ and $G \neq K_{1}, G$ has an edgecut $X$ with $|X|=2$. Define a relation $\sim$ on $E(G)$ as follows: $e \sim e^{\prime}$ if and only if $\left\{e, e^{\prime}\right\}$ is an edge-cut or $e=e^{\prime}$. It is well known that this defines an equivalence relation. Let $W=\left\{e_{1}, e_{2}, \cdots, e_{m}\right\}$ be an equivalence class and let $C_{1}, C_{2}, \cdots, C_{m}$ be the components of $G-W$.

Note that for each $i$ with $1 \leq i \leq m$, either $C_{i}=K_{1}$ or $\lambda_{2}\left(C_{i}\right)=2$, for if $C_{i}$ has an edge-cut $e^{\prime}$, then $e^{\prime}$ would have been in $W$. Also, $G /\left(C_{1} \cup C_{2} \cup \cdots \cup C_{m}\right)=$ an $m$-cycle, 
which is in $M(2)$. Note that for those $C_{i} \neq K_{1}, 2 \leq \lambda_{2}\left(C_{i}\right) \leq \bar{\lambda}_{2}\left(C_{i}\right) \leq \bar{\lambda}_{2}(G)=2$. Thus $\bar{\lambda}_{2}\left(C_{i}\right)=2$. By induction, $C_{i} \in M(2)$. Hence $G$ is obtained by replacing some vertices of an $m$-cycle by graphs in $M(2)$.

Denote $L_{2, l}=K_{2}+(l-1) K_{1}$. Let $\left[G_{1}, G_{2}\right]_{2}$ denotes the collection of all simple graphs obtained from the disjoint union of $G_{1}$ and $G_{2}$, by joining vertices in $G_{1}$ to those in $G_{2}$ with exactly 2 edges. Let $L(2, l)$ be the collection of simple graphs such that $K_{1}, L_{2, l} \in L(2, l)$, and such that a graph $G \in L(2, l)-\left\{K_{1}, L_{2, l}\right\}$ if and only if for some $G_{1}, G_{2} \in L(2, l-1), G \in\left[G_{1}, G_{2}\right]_{2}$.

Theorem 5.12 Let $G$ be a simple graph and $|V(G)| \geq l \geq 3$ and suppose $\lambda_{2}(G)=2$. Then $(l-1) \bar{\lambda}_{2}(G)=\lambda_{l}(G)$ if and only if $G \in L(2, l)$.

Proof Assume $(l-1) \bar{\lambda}_{2}(G)=\lambda_{l}(G)$. Let $X$ be a minimum edge-cut of $G$. Denote the two components by $H_{1}$ and $H_{2}$. By Theorem 5.9, either $H_{i}=K_{1}$ or $H_{i}$ satisfies Theorem 5.9(i) and Theorem 5.9(ii), for $1 \leq i \leq 2$, and so $G \in L(2, l)$.

Conversely, assume $G \in L(2, l)$. Then for some $G_{1}, G_{2} \in L(2, l-1), G \in\left[G_{1}, G_{2}\right]_{2}$. Note that $\left[G_{1}, G_{2}\right]_{2} \subseteq M(2)$, and by induction on $|V(G)|, G_{i} \in M(2)$. Thus $G \in M(2)$ and so $\bar{\lambda}_{2}(G)=\lambda_{2}(G)=2$. Thus $G$ satisfies Theorem 5.8(ii). Hence, for each value $l \geq 2$,

$$
L(2, l) \subseteq M(2)
$$

Let $\left\{X_{1}, X_{2}, \cdots, X_{l-1}\right\}$ be an arbitrarily selected minimal $l$-sequential cut of $G$. By (the above equation), $\left|X_{i}\right| \geq 2$. Since $\bar{\lambda}_{2}(G)=2,\left|X_{i}\right| \leq 2$. Thus $\left|X_{i}\right|=2$ for all $1 \leq i<l$. So $G$ satisfies Theorem 5.8(iii). By Lemma 5.7, $\lambda_{l}(G) \leq(l-1) \bar{\lambda}_{2}(G)=$ $(l-1) 2=\sum_{i=1}^{l-1}\left|X_{i}\right|$. Let $\left\{Y_{1}, Y_{2}, \cdots, Y_{l-1}\right\}$ be a minimum $l$-sequential cut of $G$. By 
Lemma 5.2, we may assume that for each $i$ with $1 \leq i \leq l-1$,

$$
Y_{i} \text { is a bond of } G_{i}=G-\cup_{j=0}^{i} Y_{j} \text {, where } Y_{0}=\emptyset \text {. }
$$

If $\sum_{i=1}^{l-1}\left|Y_{i}\right|=2(l-1)$, then every minimal $l$-sequential cut is also a minimum $l$-sequential cut; and then follows that $G$ satisfies Theorem 5.8(i)-(iii), and so ( $l$ 1) $\bar{\lambda}_{2}(G)=\lambda_{l}(G)$.

Therefore, we assume that

$$
\sum_{i=1}^{l-1}\left|Y_{i}\right|<2(l-1) .
$$

Note that by $(5.2)\left|Y_{1}\right| \geq \lambda_{2}(G)=2$.

Suppose first that $\left|Y_{1}\right|=2$. Let $H_{1}$ and $H_{2}$ be the two components of $G-Y_{1}$. By Theorem 5.9, we may assume that $H_{1}$ satisfies $(l-2) \bar{\lambda}_{2}\left(H_{1}\right)=\lambda_{l-1}\left(H_{1}\right)$. Let $\left\{Y_{2}^{\prime}, \cdots, Y_{l-1}^{\prime}\right\}$ be a minimal $(l-1)$-sequential cut of $H_{1}$. Then $\left\{Y_{1}, Y_{2}^{\prime}, \cdots, Y_{l-1}^{\prime}\right\}$ is a minimal $l$-sequential cut of $G$. By applying Theorem 5.8 to $H_{1}\left\{Y_{1}, Y_{2}^{\prime}, \cdots, Y_{l-1}^{\prime}\right\}$ is a minimum $l$-sequential cut of $G$ with $\left|Y_{i}^{\prime}\right|=\left|Y_{1}\right|=2$, for each $i$ with $2 \leq i \leq l-1$. It follows by Theorem 5.8 that $\left\{X_{1}, X_{2}, \cdots, X_{l-1}\right\}$ would be a minimum $l$-sequential cut, contrary to the assumption (5.3).

Therefore, we must have $\left|Y_{1}\right| \geq 3$. Since $G \in\left[G_{1}, G_{2}\right]_{2}$, we may assume that $Y_{1}^{\prime}=Y_{1} \cap E\left(G_{1}\right) \neq \emptyset$. For if both $Y_{1} \cap E\left(G_{1}\right)=\emptyset$ and $Y_{1} \cap E\left(G_{2}\right)=\emptyset$, then one must have $\left|Y_{1}\right|=\left|E(G)-\left(E\left(G_{1}\right) \cup E\left(G_{2}\right)\right)\right|=2$, a contradiction.

Next, we claim that $l-1 \geq 3$. For if $l=3$, then by $\left|Y_{1}\right| \geq 3$, and by $\sum_{i=1}^{2}\left|Y_{i}\right|<$ $2(3-1)$, one must have $Y_{2}=\emptyset$, a contradiction.

Now we can exclude the possibility that $G_{2}=K_{1}$. For if $G_{2}=K_{1}$, then by (5.3), $\left\{Y_{1}, Y_{2}, \cdots, Y_{l-2}\right\}$ would be an $(l-1)$-sequential cut of $G_{1}$ with $\sum_{i=1}^{l-2}\left|Y_{i}\right| \leq$ $2 l-3-\left|Y_{l-1}\right| \leq 2(l-2)$. Recall that $G_{1} \in L(2, l-1)$. Since $l-1 \geq 3$ and by induction, $G_{1}$ satisfies $(l-2) \bar{\lambda}_{2}(G)=\lambda_{l-1}(G)$. Thus by $\sum_{i=1}^{l-2}\left|Y_{i}\right| \leq 2 l-3-\left|Y_{l-1}\right| \leq 2(l-2)$, $\left\{Y_{1}, Y_{2}, \cdots, Y_{l-2}\right\}$ is a minimum $(l-1)$-sequential cut of $G_{1}$. By Theorem $5.8,\left|Y_{1}\right|=2$, contrary to the assumption that $\left|Y_{1}\right| \geq 3$. 
Thus both $G_{1}$ and $G_{2}$ are not $K_{1}$. Now define $Y_{i}^{\prime}=Y_{i} \cap E\left(G_{1}\right)$ and $Y_{i}^{\prime \prime}=Y_{i} \cap E\left(G_{2}\right)$ for each $i$ with $1 \leq i \leq l-1$. Since $G_{1} \in L(2, l-1) \subset M(2)$ and by (5.2), either $\left|Y_{i}^{\prime}\right|=0$ or $\left|Y_{i}^{\prime}\right| \geq 2$. Similarly, we conclude that either $\left|Y_{i}^{\prime \prime}\right|=0$ or $\left|Y_{i}^{\prime \prime}\right| \geq 2$.

Hence we may assume that there are $p_{1}$ nonempty sets in $Y_{1}^{\prime}, Y_{2}^{\prime}, \cdots Y_{l-1}^{\prime}$ and $p_{2}$ nonempty sets in $Y_{1}^{\prime \prime}, Y_{2}^{\prime \prime}, \cdots Y_{l-1}^{\prime \prime}$. Since $\left\{Y_{1}, \cdots, Y_{l-1}\right\}$ is an $l$-sequential cut of $G$, $p_{1}+p_{2} \geq l-1$.

If $p_{1}=l-1$, then $\left\{Y_{1}^{\prime}, Y_{2}^{\prime}, \cdots Y_{l-2}^{\prime}\right\}$ is an $(l-1)$-sequential cut of $G_{1}$, and so $\sum_{i=1}^{l-2}\left|Y_{i}^{\prime}\right| \geq 2(l-2)$, by induction. Since $\left|Y_{1}\right| \geq 3$, either $\sum_{i=1}^{l-2}\left|Y_{i}^{\prime}\right|>2(l-2)$ or $\left|Y_{1}^{\prime \prime}\right| \geq 1$. This together with $\left|Y_{l-1}\right| \geq 1$, yields $\sum_{i=1}^{l-1}\left|Y_{i}\right| \geq 2(l-1)$, contrary to (5.3).

Therefore we may assume that $p_{1} \leq l-2$ and $p_{2} \leq l-2$. Since $G_{j} \in L(2, l-1) \subseteq$ $L\left(2, p_{j}+1\right)$, by induction, $\sum_{i=1}^{p_{j}}\left|Y_{i}^{\prime}\right| \geq 2 p_{j}$. It follows that $\sum_{i=1}^{l-2}\left|Y_{i}\right|=\sum_{j=1}^{2} \sum_{i=1}^{p_{j}}\left|Y_{i}^{\prime}\right| \geq$ $2\left(p_{1}+p_{2}\right) \geq 2(l-1)$, contrary to $(5.3)$.

These contradictions indicate that the arbitrarily selected minimal $l$-sequential cut $\left\{X_{1}, \cdots X_{l-1}\right\}$ must be minimum, and so $G$ satisfies $(l-1) \bar{\lambda}_{2}(G)=\lambda_{l}(G)$. 


\section{Chapter 6}

\section{Minimally $(k, k)$-edge connected graphs}

\subsection{Elementary properties of minimally $(k, k)$-edge connected graphs}

Let $k \geq 3$ be an integer. In this section we consider connected graphs having edge connectivity greater than 1 . If $\lambda>1$ for a graph $G$, then $G$ has no cut-edges. If $G$ is a connected graph without a cut-edge, then $G-e$ is a connected graph for any edge $e$ of $G$.

Property 6.1 Let $G$ be a connected graph without cut-edges. The following are equivalent:

(i) $\mathrm{G}$ is minimally $(k, k)$-edge connected graph.

(ii) for each edge $e$ of $G, G-e$ has at least $k-1$ cut-edges $e_{1}, e_{2}, \ldots, e_{k-1}$.

Proof: Let $G$ be a minimally $(k, k)$-edge connected graph. Since $G$ is minimally 
$(k, k)$-edge connected $\lambda_{k}(G)=k$ and $\lambda_{k}(G-e)<k$, for any edge $e$ of $G$. Thus $\lambda_{k}(G-e)=k-1$ for any edge $e \in E(G)$. Thus there are $k-1$ edges $a_{1}, a_{2}, \ldots, a_{k-1}$ of $G-e$ whose removal gives $k$ components. In order to attain this the removal of any edge $a_{i}, 1 \leq i \leq k-1$ should increase the number of components by one. Therefore, for any edge $a_{i}, 1 \leq i \leq k-1$ of $E(G-e),(G-e)-a_{i}$ has two components. Hence, for all $1 \leq i \leq k-1, a_{i}$ edges are cut-edges of $G-e$.

Conversely, $G-e$ has $k-1$ cut-edges $e_{1}, e_{2}, \ldots, e_{k-1}$ for any edge $e$ of $E(G)$. Thus for any edge $e \in E(G), \lambda_{k}(G-e)=k-1$ and $\lambda_{k}(G)=k$. Therefore $G$ is minimally $(k, k)$-edge connected.

Theorem 6.2 Let $G$ be a connected graph without cut edges. If $G$ is minimally $(k, k)$-edge connected then it is minimally $(2,2)$-edge connected.

Proof: Let $G$ be a minimally $(k, k)$-edge connected graph. Then $\lambda_{k}(G)=k$ and for any edge $e$ of $G, \lambda_{k}(G-e)=k-1$. We have $\lambda_{k}(G) \geq \frac{k}{2} \lambda_{2}(G)$ by equation 4.1 . Thus $2 \geq \lambda_{2}(G)$. Therefore $\lambda_{2}(G)=2$, or $\lambda_{2}(G)=1$. Since $G$ has no cut edges $\lambda_{2}(G)>1$. Thus $\lambda_{2}(G)=2$. Since $\lambda_{k}(G-e)=k-1, k-1 \geq \frac{k}{2} \lambda_{2}(G-e)$. Thus $\frac{2(k-1)}{k} \geq \lambda_{2}(G-e)$ and this gives $\lambda_{2}(G-e)=1$ for any edge $e$ of $G$. Therefore $G$ is minimally $(2,2)$-edge connected.

Property 6.3 Let $G$ be a connected graph without cut edges. If $G$ is minimally $(k, k)$-edge connected, then for any subset $Y$ of $V(G)$ such that $|Y| \geq k$, the $k$-edgeconnectivity of the induced subgraph $G(Y)$ is not greater than $k$. Furthermore, if $G(Y)$ is $(k, k)$-edge connected, then it is minimally $(k, k)$-edge connected. 
Proof: Let $G$ be a minimally $(k, k)$-edge connected graph. By contradiction assume that there is a subset $Y$ of $V(G)$, such that the $k$-edge-connectivity of the induced subgraph $G(Y)$ is greater than $k$. That is $\lambda_{k}(G(Y))>k$. Thus $\lambda_{k}\left(G(Y)-T_{1}\right)=k$ for some edge set $T_{1}$ of $E(G(Y))$. Since $\lambda_{k}(G)=k$, there is an edge subset $T_{2}$ of $E(G)$ such that $\left|T_{2}\right|=k$ and $G-T_{2}$ has $k$ components. Thus there is an edge $e \in\left(T_{1}-T_{2}\right)$. Therefore $\lambda_{k}(G-e)=k$. This contradicts the minimality. Thus for any subset $Y$ of $V(G)$, the $k$-edge connectivity of $G(Y)$ is not greater than $k$. Now suppose $G(Y)$ is $(k, k)$-edge connected. If $G(Y)$ is not minimally $(k, k)$-edge connected there is an edge $e^{\prime}$ of $G(Y)$ such that $\lambda_{k}\left(G(Y)-e^{\prime}\right)=k$. This gives $\lambda_{k}\left(G-e^{\prime}\right)=k$ contradicting the minimality.

Let $G_{1}$ and $G_{2}$ be two graphs with disjoint vertex sets. Let $G_{1} \oplus G_{2}$ denote the graph obtained from $G_{1}$ and $G_{2}$ by identification of $v_{1} \in V\left(G_{1}\right)$ and $v_{2} \in V\left(G_{2}\right)$.

Property 6.4 Let $G_{1}$ and $G_{2}$ be two connected graphs without cut edges, and let $G=G_{1} \oplus G_{2}$. Then the following are equivalent.

(i) Both $G_{1}$ and $G_{2}$ are minimally $(k, k)$-edge connected.

(ii) $G$ is minimally $(k, k)$-edge connected.

Proof: Let $G_{1}$ and $G_{2}$ be two minimally $(k, k)$-edge connected graphs without cut edges and $V\left(G_{1}\right) \cap V\left(G_{2}\right)=\emptyset$. Let $G$ be the graph obtained from $G_{1}$ and $G_{2}$ by identification of $v_{1} \in V\left(G_{1}\right)$ and $v_{2} \in V\left(G_{2}\right)$. Thus $G=G_{1} \oplus G_{2}$. Let $X$ be an $\lambda_{k}$ edge cut of $G$. That is $\lambda_{k}(G)=|X|$. Let $X \cap E\left(G_{i}\right)=X_{i}$ for an integer $i$ such that $1 \leq i \leq 2$. Then $|X|=\left|X_{1}\right|+\left|X_{2}\right|$, and each $X_{i}$ is an edge cut of $G_{i}$. Let $G_{i}-X_{i}$ has $l_{i}$ components for each $i \in\{1,2\}$. Since $G_{i}-X_{i}$ has $l_{i}$ components $\left|X_{i}\right| \geq l_{i}$. Thus $k=l_{1}+l_{2} \leq\left|X_{1}\right|+\left|X_{2}\right|$. Therefore $|X| \geq k$. Thus $\lambda_{k}(G)=k$. For any $e \in E(G)$, 
either $e \in E\left(G_{1}\right)$ or $e \in E\left(G_{2}\right)$. Therefore $\lambda_{k}(G-e)=k-1$ for any edge $e \in E(G)$. Thus $G$ is minimally $(k, k)$-edge connected.

Conversely let $G_{1}$ and $G_{2}$ be two connected graphs without cut edges, and let $G=G_{1} \oplus G_{2}$ be a minimally $(k, k)$-edge connected graph. Then each $\left(G_{i}\right)$ is an induced subgraph of $G$ and by Property $6.3 G_{i}$ is a minimally $(k, k)$-edge connected graph for $i=\{1,2\}$.

What Property 6.4 indicates is that to study the structure of minimally $(k, k)$-edge connected graphs without cut edges, it suffices to study the structure of 2-connected minimally $(k, k)$-edge connected graphs. Motivated from this view point and from the similar concepts in $[\mathrm{CC}]$, we present the following definitions.

Let a $k$-necklace be a 2-connected minimally $(k, k)$-edge connected simple graph. When $k=2, k$-necklace is a necklace. Thus $k$-necklace is a generalization of a necklace.

Lemma 6.5 A cycle of length $\geq k$ is a $k$-necklace.

Proof: Let $G$ be cycle of length $\geq k$. Then $\lambda_{k}(G)=k$ and $\lambda_{k}(G-e)=k-1$ for any $e \in E(G)$. Thus $G$ is a minimally $(k, k)$-edge connected graph.

A graph $G$ is $k$-extensible between $x$ and $y$ if, $x$ and $y$ being two distinct vertices of $G$, the graph $G_{x y}^{\alpha_{1}, \alpha_{2}, \ldots, \alpha_{k-1}}$ obtained from $G$ by adding $k-1$ new distinct vertices $\alpha_{1}, \alpha_{2}, \ldots, \alpha_{k-1}$ and the $k$ new edges $x \alpha_{1}, \alpha_{1} \alpha_{2}, \ldots, \alpha_{k-1} y$, is minimally $(k, k)$-edge connected. 
Lemma 6.6 Let $G$ be a graph with two distinct vertices $x$ and $y$ and the edge $x y$. That is the graph $G$ is the edge $x y$. Then $G$ is $k$-extensible between $x$ and $y$.

Proof: Let $G$ be a graph with two distinct vertices $x$ and $y$ and the edge $x y$. Then the graph $G_{x y}^{\alpha_{1}, \alpha_{2}, \ldots, \alpha_{k-1}}$ is a $k$-cycle and it is a minimally $(k, k)$-edge connected graph (by Lemma 6.5). Thus $G$ is $k$-extensible between $x$ and $y$.

Lemma 6.7 Let $G$ be a cycle. For any edge $e=a b$ of $G, G-e$ is $k$-extensible between $a$ and $b$.

Proof: Let $G$ be a cycle and $e=a b$ be an edge of $G$. Let $H=G-e$. Then the graph $H_{a b}^{\alpha_{1}, \alpha_{2}, \ldots, \alpha_{k-1}}$ is a cycle of length $\geq k$. Thus by Lemma $6.5, G-e$ is $k$-extensible between $a$ and $b$.

A graph $G$ is called an $E_{k}$-chain if it can be represented by $a_{0} G_{1} a_{1} G_{2} a_{2} \cdots a_{l-1} G_{l} a_{l}$ where all of the following properties are satisfied:

(i) $l \geq k-1$.

(ii) $\forall i=1,2, \ldots, l: G_{i}$ is either an edge or a $k$ - necklace.

(iii) If there is a $G_{i}$ which is an edge then there exists at least $k-1, G_{j}$ 's, $1 \leq j \leq l$, such that there are at least $k-1$ edges.

(iv) $\forall i=1,2, \ldots, l-1: V\left(G_{i}\right) \cap V\left(G_{i+1}\right)=a_{i}$.

(v) $\forall i=1,2, \ldots, l-2$ and $\forall j=i+2, \ldots, l: V\left(G_{i}\right) \cap V\left(G_{j}\right)=\emptyset$.

Each $G_{i}$ is called a component of the $E_{k}$-chain. For $k=2, E_{k}$-chain is an $E$-chain. 
Thus $E_{k}$-chain is a generalization of $E$-chain.

Lemma 6.8 Let $G$ be an $E_{k}$-chain and is represented by $a_{0} G_{1} a_{1} G_{2} a_{2} \cdots a_{l-1} G_{l} a_{l}$. Then the following are equivalent.

(i) $G+a_{0} a_{l}$ is a $k$-necklace.

(ii) One of the $G_{i}, 1 \leq i \leq l$ is an edge.

Proof: Let $G$ be an $E_{k}$-chain and is represented by $a_{0} G_{1} a_{1} G_{2} a_{2} \cdots a_{l-1} G_{l} a_{l}$. Let $G+a_{0} a_{l}$ be a $k$-necklace. Then $G+a_{0} a_{l}$ is minimally $(k, k)$-edge connected, by the definition of a $k$-necklace. By Property $6.1, G+a_{0} a_{l}-a_{0} a_{l}=G$ has at least $k-1$ cut edges. Since $G$ is an $E_{k}$-chain if $G$ has one cut edge then it has at least $k-1$ cut edges. Thus one of the $G_{i}$ is an edge.

Conversely let one of the $G_{i}$ be an edge. Then by the definition of $E_{k}$-chain at least $k-1, G_{i}$ 's, are edges. Thus for any edge $e \in E\left(G+a_{0} a_{l}\right), G-e$ has at least $k-1$ cut edges, since $e$ is an edge in a $k$-necklace $G_{i}$ or $e$ is an edge $G_{i}$, or $e$ is the edge $a_{0} a_{l}$. Thus $G+a_{0} a_{l}$ is a $k$-necklace, by Property 6.1 .

\subsection{Characterization of minimally $(k, k)$-edge con- nected graphs without cut edges}

Lemma 6.9 Let $G$ be a 2-connected graph. The following are equivalent.

(i) $G$ is a $k$-necklace.

(ii) For any edge $e=a b$ of $E(G), G-e$ is an $E_{k}$-chain $a G_{1} a_{1} G_{2} a_{2} \cdots a_{l-1} G_{l} b$ such that there are at least $k-1$ of the $G_{j}$ 's are edges. 
Proof: Let $G$ be a $k$-necklace. For any edge $e=a b \in E(G), G-e$ has at least $k-1$ cut edges (Property 6.1 ). Thus $G-e$ can be represent as an $E_{k}$-chain $a G_{1} a_{1} G_{2} a_{2} \cdots a_{l-1} G_{l} b$ such that there are at least $k-1$ of the $G_{j}$ 's are edges.

Conversely, assume Lemma 6.9(ii). Then by Lemma $6.8, G$ is a $k$-necklace.

Lemma 6.10 Let $G$ be $k$-necklace and $x, y$ be two distinct vertices of $G$. The following are equivalent.

(i) $G$ is $k$-extensible between $x$ and $y$.

(ii) for each edge $e$ of $E(G)$, the $E_{k}$-chain $G-e$ can be described by $a_{0} G_{1} a_{1} G_{2} a_{2} \cdots a_{l-1} G_{l} a_{l}$, such that for some $i$ and $j, 1 \leq i \leq j \leq l, x \in V\left(G_{i}\right), y \in V\left(G_{j}\right)$, and

there are at least $k-1, G_{h}$ edges where $h \notin\{i, i+1, \ldots, j\}$.

Proof: Let $G$ be a $k$-necklace. Assume Lemma 6.10(i). By Lemma 6.9(ii), for any edge $e \in E(G), G-e$ is an $E_{k}$-chain $a_{0} G_{1} a_{1} G_{2} a_{2} \cdots a_{l-1} G_{l} a_{l}$. As $x, y \in V(G)=$ $V(G-e)$, we may assume that for some $i$ and $j 1 \leq i \leq j \leq l, x \in V\left(G_{i}\right), y \in V\left(G_{j}\right)$. By contradiction assume further that (6.1) fails. Then $G_{x y}^{\alpha_{1}, \alpha_{2}, \ldots, \alpha_{k-1}}-e$ has at most $k-2$ cut edges. Thus by Property $6.1 G_{x y}^{\alpha_{1}, \alpha_{2}, \ldots, \alpha_{k-1}}-e$ is not minimally $(k, k)$-edge connected. This contradicts the definition of $k$-extensibility between $x$ and $y$.

Conversely, assume Lemma 6.10(ii). Let us consider the $E_{k}$-chain of $G$ associated with $e: a_{0} G_{1} a_{1} G_{2} \cdots a_{l-1} G_{l} a_{l}$. Suppose that $x \in V\left(G_{i}\right), y \in V\left(G_{j}\right), i \leq j$. By contradiction assume that $G_{x y}^{\alpha_{1}, \alpha_{2}, \ldots, \alpha_{k-1}}$ is not minimally $(k, k)$-edge connected. $G$ is $(k, k)$-edge connected and so is $G_{x y}^{\alpha_{1}, \alpha_{2}, \ldots, \alpha_{k-1}}$. Thus there exists an edge $e$ of $E\left(G_{x y}^{\left.\alpha_{1}, \alpha_{2}, \ldots, \alpha_{k-1}\right)}\right)$ such that $G_{x y}^{\alpha_{1}, \alpha_{2}, \ldots, \alpha_{k-1}}-e$ still remains $(k, k)$-edge connected. But this implies $e \neq x \alpha_{1}, e \neq \alpha_{1} \alpha_{2}, \cdots, e \neq \alpha_{k-1} y$, and thus $e \in E(G)$. By Lemma 
6.10(ii), there are at least $k-1, G_{h}$ edges with $h \notin\{i, i+1, \ldots, j\}$. These $k-1$ edges are clearly cut edges of $G_{x y}^{\alpha_{1}, \alpha_{2}, \ldots, \alpha_{k-1}}-e$, which is a contradiction.

Corollary 6.11 A cycle is $k$-extensible between $x$ and $y$ if and only if the distance between $x$ and $y$ is greater or equal to $k$.

Proof: Let $G$ be a cycle and $x$, and $y$ be two vertices of $G$. Let $P$ be a path between $x$ and $y$. Then the distance between $x$ and $y$ is lowest size of $P$, and is denoted by $\min (m(P))$.

Suppose $G$ is $k$-extensible between $x$ and $y$. Then $G_{x y}^{\alpha_{1}, \alpha_{2}, \ldots, \alpha_{k-1}}-e$ has at least $k-1$ cut edges for any $e \in E(G)$. Therefore for $e \in P, m(P-e) \geq k-1$. Thus the distance between $x$ and $y$ must be greater or equal to $k$.

Conversely assume the distance between $x$ and $y$ is greater or equal to $k$. Then for any $e \in E(G), G_{x y}^{\alpha_{1}, \alpha_{2}, \ldots, \alpha_{k-1}}-e$ has at least $k-1$ cut edges. Thus $G$ is $k$-extensible between $x$ and $y$.

Lemma 6.12 Let $G=a_{0} G_{1} a_{1} G_{2} a_{2} \cdots a_{l-1} G_{l} a_{l}, l \geq k$, denote an $E_{k}$-chain. The following are equivalent.

(i) $G$ is $k$-extensible between $a_{0}$ and $a_{l}$.

(ii) for each $i=1,2, \ldots, l, G_{i}$ is $k$-extensible between $a_{i-1}$ and $a_{i}$.

Proof: Suppose first that $G$ is $k$-extensible between $a_{0}$ and $a_{l}$, and that $G_{i}$ is not $k$-extensible between $a_{i-1}$ and $a_{i}$. Thus $G_{i}$ is not an edge and hence it is a $k$-necklace. By Lemma 6.10, there exists $e \in E\left(G_{i}\right)$ such that there are at most $k-2$ cut edges 
of $G_{i}-e$ not lying between $a_{i-1}$ and $a_{i}$ on the $E_{k}$-chain $G_{i}-e$. It is then clear that $G_{a_{0} a_{l}}^{\alpha_{1}, \alpha_{2}, \ldots, \alpha_{k-1}}-e$ has at most $k-2$ cut edge. This contradicts $G$ is $k$-extensible between $a_{0}$ and $a_{l}$.

Suppose conversely that for each $i, i=1,2, \cdots, l, G_{i}$ is $k$-extensible between $a_{i-1}$ and $a_{i}$, but that $G$ is not $k$-extensible between $a_{0}$ and $a_{l}$. Hence $G_{a_{0} a_{l}}^{\alpha_{1}, \alpha_{2}, \ldots, \alpha_{k-1}}$ is not minimally $(k, k)$-edge connected. But $G_{a_{0} a_{l}}^{\alpha_{1}, \alpha_{2}, \ldots, \alpha_{k-1}}$ is $(k, k)$-edge connected and hence there exists $e \in E\left(G_{a_{0} a_{l}}^{\alpha_{1}, \alpha_{2}, \ldots, \alpha_{k-1}}\right)$ such that $G_{a_{0} a_{l}}^{\alpha_{1}, \ldots, \alpha_{2}, \ldots, \alpha_{k-1}}-e$ is $(k, k)$-edge connected. Clearly $e \neq a_{0} \alpha_{1}, e \neq \alpha_{1} \alpha_{2}, \cdots e \neq \alpha_{k} a_{l}$, and hence $e \in E\left(G_{i}\right)$ for some $i$. Since $G_{a_{0} a_{l}}^{\alpha_{1}, \ldots, \alpha_{2-1}, \alpha_{k-1}}-e$ is $(k, k)$-edge connected, $G_{i}$ is not an edge; thus it is a $k$-necklace. But then $G_{i}-e$ is an $E$-chain by Lemma 6.9 and there are at most $k-2$ cut edges of $G_{i}-e$ do not lie between $a_{i-1}$ and $a_{i}$ and thus $G_{i}$ is not $k$-extensible between $a_{i-1}$ and $a_{i}$, a contradiction.

Lemma 6.13 Let $G$ be a $k$-necklace, $e$ an edge in $G$, and $a_{0} G_{1} a_{1} G_{2} \cdots G_{l} a_{l}$ the $E_{k}$-chain $G-e$. Each $E_{k}$-chain $a_{i-1} G_{i} a_{i} G_{i+1} a_{i+1} \cdots G_{i+h} a_{i+h}, i=1,2, \cdots, l-1$, $h=1,2, \cdots, l-i$, is $k$-extensible between $a_{i-1}$ and $a_{i+h}$.

Proof: By contradiction, suppose that for some $i, a_{i-1} G_{i} a_{i} G_{i+1} a_{i+1} \cdots G_{i+h} a_{i+h}$, is not $k$-extensible between $a_{i-1}$ and $a_{i+h}$. By Lemma 6.12, there exists $q, 0 \leq q \leq h$, such that $G_{i+q}$ is not $k$-extensible between $a_{i+q-1}$ and $a_{i+q}$. By Lemma 6.10, there exists an edge $f$ in $G_{i+q}$ that does not satisfy (6.1). Therefore there are at most $k-2$ cut edges in $G_{i+q}-f$ not lying between $a_{i+q-1}$ and $a_{i+q}$. But then $G-f$ has no $k-1$ cut edges and hence $G$ is not a $k$-necklace, a contradiction.

Let $F_{k 0}$ denote the family of graphs obtained by the following. 
$\left(F_{0} 1\right)$ Every cycle of length $\geq k$ belongs to $F_{k 0}$.

$\left(F_{0} 2\right)$ A graph $G \in F_{k 0}$ if and only if for some integer $l \geq k$, there are $l$ vertex-disjoint graphs $G_{1}, G_{2}, \cdots, G_{l}$ such that

(a) $\forall i$ with $1 \leq i \leq l, G_{i} \in F_{k 0}$ or $G_{i} \cong K_{2}$, and at least $k$ of the $G_{i}$ 's are isomorphic to $K_{2}$;

(b) there exist two distinct vertices $\left\{x_{i}, y_{i}\right\} \subset V\left(G_{i}\right)$ such that $G_{i}$ is $k$-extensible between $x_{i}$ and $y_{i}$ for each $i$ with $1 \leq i \leq l$; and such that $G$ can be obtained from these $G_{i}$ 's by identifying $x_{1}$ and $y_{l}$, and for $1 \leq i \leq l-1$, identifying $y_{i}$ and $x_{i+1}$.

Theorem 6.14 The family of the $k$-necklaces is $F_{k 0}$.

Proof: First, we show that every necklace belongs to $F_{k 0}$. Let $G$ be a necklace. If $G$ is a cycle, then by Lemma $6.5, G$ must be a cycle of length at least $k$, and so by $\left(F_{0} 1\right), G \in F_{k 0}$. Assume that $G$ is not a cycle. Pick $e=a b \in E(G)$. By Lemma 6.9(ii), $G-e$ is an $E_{k}$-chain $a G_{1} a_{1} G_{2} a_{2} \cdots a_{l-1} G_{l} b$ such that $l \geq k$ and at least $k-1$ of the $G_{j}$ 's are edges. By Lemma 6.13 , this $E_{k}$-chain satisfies $\left(F_{0} 1\right)$, and so $G \in F_{k 0}$.

Conversely, let $G \in F_{k 0}$. We shall argue by induction on $|V(G)|$ to show that $G$ is a necklace. If $G$ is a cycle, then by $\left(F_{0} 1\right), G$ has length at least $k$, and so by Lemma $6.5, G$ is a necklace. Hence we assume $G$ is obtained via $\left(F_{0} 2\right)$.

Note that since $x_{i} \neq y_{i}$, for each $i$ in $\left(F_{0} 2\right), G$ is 2-connected. Also, by induction, each $G_{i}$ in $\left(F_{0} 2\right)$ is either isomorphic to a $K_{2}$ or is a necklace. For any $e \in E(G)$, if $e$ is one of the $G_{i}$ 's which is isomorphic to a $K_{2}$, then by (a) of $\left(F_{0} 2\right)$, there are at least $k$ such $G_{i}$ 's. If $e \in E(G)$ for some $G_{i} \neq K_{2}$, then since $G_{i}$ is a necklace and is $k$-extensible between $x_{i}$ and $y_{i}$, by Lemma 6.10 , there are at least $k-1$ edges $G_{h}$ 
edges which are not lying between $x_{i}$ and $y_{i}$ in the $E_{k}$-chain $G_{i}-e$. Thus for any edge $e \in E(G), G-e$ is an $E_{k}$-chain having at least $k-1$ of the $G_{j}$ ' edges. Hence by Lemma 6.9(ii), $G$ is a necklace.

Let $F_{k}$ denote the family of graphs obtained by the following.

(F1) $F_{k 0} \subset F_{k}$.

(F2) A graph $G \in F_{k}$ if and only if either $G \in F_{k 0}$, or there are two edge disjoint proper subgraphs $G_{1}$ and $G_{2}$ of $G$ with $\left|V\left(G_{1}\right) \cap V\left(G_{2}\right)\right|=1$ and $G=G_{1} \cup G_{2}$ such that $G_{1}, G_{2} \in F_{k}$.

Theorem 6.15 The family of minimally $(k, k)$-edge connected graphs without cut edges is $F_{k}$.

Proof: Let $G$ be a minimally $(k, k)$-edge connected graph without cut edges. If $G$ is also 2-connected then $G \in F_{k 0} \subset F_{k}$, by Theorem 6.14. If $G$ has a cut vertex, then by Property $6.4 G \in F_{k}$, by (F2). Hence every minimally $(k, k)$-edge connected graph without cut edges is in $F_{k}$.

Conversely, let $G \in F_{k}$. If $G \in F_{k 0}$, then by Theorem $6.14, G$ is a minimally $(k, k)$-edge connected graph without cut edges. If $G$ is obtained via $(\mathrm{F} 2)$, then by Property $6.4, G$ is also a minimally $(k, k)$-edge connected graph without cut edges. This proves the theorem. 
Chapter 7

\section{Bibliography}




\section{Bibliography}

[AH] A.T. Amin and S.L. Hakimi, Graphs with given connectivity and independence number, IEEE Trans.Circuit Theory, CT-20 (1973), 2-10.

[Bar] C.A. Barefoot, 4-connected Halin graphs are 2-Hamiltonian connected Ars Combinatoria 35-A (1993), 109-128.

[BH] L.W. Beineke and F. Harary, The connectivity function of a graph, Matheatika 14 (1967), 197-202.

[Ber] C. Berge, Theories des Graphes et ses Applications, Dunod, Paris, (1958).

[BB] J.C. Bermond and B. Bollobás, The diameter of graphs - A survey, Congr. Numerantium 32 (1981) 3-27.

[BBPP] J.C. Bermond, J. Bond, M. Paoli and C. Peyrat, Graphs and interconnection networks: Diameter and vulnerability, in Surveys in Combinatorics (K. Lloyd, ed.) London Math. Soc. Lecture Notes Series 82 (1983) 1-30.

[Boe] F.T. Boesch, Synthesis of reliable networks - A survey, IEEE transactions on reliability, vol. r-35, 3 (1986), 240 - 246.

[BoC] F.T. Boesch and S.Chen, A generalization of line connectivity and optimally invulnerable graphs, SIAM J. Appl. Math 34 (1978), 657-665. 
[BF1] F.T. Boesch and A.P. Felzer, A general class of invulnerable graphs, Networks, 2 (1970), 261-283.

[BF2] F.T. Boesch and A.P. Felzer, On the minimum $m$ degree vulnerability criterion, IEEE Trans. Circuit Theory, CT-18 (1971), 224-228.

[BM] F.T. Boesch and J.A.M. McHugh, An edge extremal result for subcohesion. J. Combinatorial Theory Series B 38 (1985) 1-7.

[BTh] F.T. Boesch and R.E. Thomas, On graphs of invulnerable communication nets, IEEE Trans. Circuit Theory, CT-17 (1970), 183-192.

[BT] F.T. Boesch and R. Tindell, Circulants and their connectivities J. Graph Theory 8 (1984) 487-499

[BW1] F.T. Boesch and J.F. Wang, Reliable circulant networks with minimum transmission delay, IEEE Trans. Circuits Syst. CAS-32 (1985) 12861291.

[BW2] F.T. Boesch and J.F. Wang, Super line-connectivity properties of circulant graph, SIAM J. Algebraic and Discrete Methods 7 (1986) 89-98.

[Bol] B. Bollobás, Extremal Graph Theory, Academic Press, London (1978).

[BrC] R.A. Brualdi and J. Csima, A note on vertex- and edge-connectivity, Bull. Inst. Comb. Appl. 2 (1991), 67-70.

[Ca1] L. Caccetta, Extremal graphs with given diameter and connectivity, Ann. N.Y. Acad. Sci. 328 (1979) 76-94.

[Ca2] L. Caccetta, Graph theory in network design and analysis, Recent studies in graph theory, ed. V.R. kulli, (1989) by Vishwa International publications. 
[Cha] G. Chartrand, A graph-theoretic approach to a communications problem, SIAM J. Appl. Math 14 (1966), 778-781.

[CH] G. Chartrand and F. Harary, Graphs with prescribed connectivities, Theory of Graphs, Academic Press, New York (1968), 61-63.

[CKLL] G. Chartrand, S.F. Kapoor, L. Lesniak and D.R. Lick, Generalized connectivity in graphs, Bull. Bombay Math. Colloq. 2 (1984), 1-6.

[CKK] G. Chartrand, S.F. Kapoor, H.V. Kronk, A sufficient condition for $n$ connectedness of graphs, Mathematika 15 (1968), 51-52.

[CL] G. Chartrand and L. Lesniak, Graphs and Digraphs. Chapman \& Hall, (1996).

[CC] G. Chaty and M. Chein, Minimally 2-edge connected graphs J. Graph Theory 3 (1979), 15-22.

[DOS] D.P. Day, O.R. Oellermann, H.C. Swart, On the l-connectivity function of caterpillars and complete multipartite graphs, JCMCC 10 (1991), 183192.

[Dir] G.A. Dirac, Minimally 2-connected graphs, J.Reine Angew. Math., 228 (1967), 204-216.

[FF] H. Frank, I. Frisch, Communication, transmission and transportation networks, Addison-Wesley (1971).

[Gal] Galil, Zvi, Italiano, Giuseppe, Maintaining the 3-edge-connected components of a graph on-line SIAM- J. -Comput. 22 (1993) no 1, 11 - 28.

[Go1] D.L. Goldsmith, On the second-order edge connectivity of a graph, Congressus Numerantium 29 (1980), 479-484. 
[Go2] D.L. Goldsmith, On the $n$-th order connectivity of a graph, Congressus Numerantium 32 (1981), 375-382.

[Go3] D.L. Goldsmith, The functional edge-connectivity of a graph, J. of Combinatorics-Information and System Sciences 17 (1992), no 3-4, 183193.

[GE] D.L. Goldsmith and R.C. Entringer, A sufficient condition for equality of edge-connectivity and minimum degree of a graph, J. Graph Theory $\mathbf{3}$ (1979), 251-255.

[GMF] D.L. Goldsmith, B. Manvel and V. Faber, Separation of graphs into three components by the removal of edges, J. Graph Theory 4 (1980), 213-218.

[GW] D.L. Goldsmith and A.T. White, On graphs with equal edge-connectivity and minimum degree, Discrete Math 23 (1978), 31-36.

[HP] M. Habib and B. Peroche, A constrction method for minimally k-edgeconnected graphs, Annals of Discrete Mathematics 9 (1980), 199-204.

[Hak] S.L. Hakimi, On an algorithm for construction of the least vulnerable communication network on a graph with maximum connectivity, IEEE Trans. Circuit Theory CT-16 (1969) 229-230.

[Hal] R. Halin, A theorem on n-connected graphs, J. Comcinatorial Theory 7 (1969), 150-154.

[Har] F. Harary, The maximum connectivity of a graph, Proc. Nat. Acad. Sci. 48 (1962), 1142-1146.

[Hed] B. Hedman, A sufficient condition for n-short-connectedness, Math. Mag. 47 (1974), 156-157. 
[HLX] K.P. Hennayake, H.-J. Lai and L. Xu, The strength and the l-edge connectivity of a graph, Bulletin of the Inst. Comb. Asso., accepted.

[HU] T.C. Hu, Integer Programming and Network Flows, Addison-Wesley, Reading, MA, 1969.

[Jol] J.-L. Jolivet, Sur les puissances des graphed connexes, C.R. Acad. Sci. Paris Ser. I Math., 272 (1971), 107-109.

[Lai] H.-J. Lai, The size of strength-maximal graphs, J. graph theory, 14 (1990), 187-197.

[Les] L. Lesniak, Results on the edge-connectivity of graphs, Discrete Math., 8 (1974), 351-354.

[Li1] D.R. Lick, Critically and minimally n-connected graphs, The many facets of graph theory, Springer-Verlag, (1969), 199-205.

[Lic] D.R. Lick, Minimally n-line connected graphs, J. Reine Angew. Math., 252 (1972), 178-182.

[Lon] J.Q. Longyear, Regular d-valent graphs of girth 6 and $2\left(d^{2}-d+1\right)$ vertices, J. Combinatorial Theory, 9 (1970), 420-422.

[Mad1] W. Mader, Minimale n-fach kantenzusammenhängende Graphen, Math. Ann., 191 (1971), 21-28.

[Mad2] W. Mader, A reduction method for edge-connectivity in graphs, Annals of Discrete Mathematics 3 (1978), 145-164.

[Mad3] W. Mader, Connectivity and edge-connectivity in finite graphs, Surveys in Combinatorics, London Math. Soc. Lecture Note Series 38 Cambridge Univ. press, Cambridge (1979) 66-95. 
[Mad4] W. Mader, On vertices of degree $n$ in minimally $n$-connected graphs and digraphs Combinatorics (1996), 423-449.

[Mao] C. Mao-chen, The number of vertices of degree $k$ in a minimally $k$-edgeconnected graph, J. Combinatorial Theory (B) 30 (1993), 225-239.

[Ma1] D.W. Matula, The cohesive strength of graphs, Washington University, Department of Applied Mathematics and Computer Science, Report AM-68-9, 1968.

[Ma2] D.W. Matula, The cohesive strength of graphs, The Many Facets of Graph Theory, Springer-Verlag, Berlin (1969) 215-221.

[Ma3] D.W. Matula, $K$-components, clusters, and slicings in graphs, SIAM J. Appl. Math. 22 (1972) 459-480.

[Ma4] D.W. Matula, Subgraph connectivity numbers of a graph, Theory and Applications of Graphs, Springer-Verlag, Berlin (1978) 371-383.

[Ma5] D.W. Matula, Determining edge connectivity in $O(n m)$, 28th Annual Symposium on Foundation of Computer Sciences (1987), 249-251.

[MS1] S. Maurer and P.J. Slater, On k-critical, $n$-connected graphs*, Discrete Mathematics 20 (1977), 255-262.

[MS2] S. Maurer and P.J. Slater, On k-minimally n-edge-connected graphs, Discrete Mathematics 24 (1978), 185-195.

[Mur] U.S.R. Murty, Extremal nonseparable graphs of diameter 2, in Proof Techniques in Graph Thery (ed. F. Harary), Academic Press, New York, (1969) pp. 111- 118. 
[Oe1] O.R. Oellermann, Generalized connectivity in graphs, Doctoral Dissertation, Western Michigan University,Michigan, 1986.

[Oe2] O.R. Oellermann, A note on the l-connectivity function of a graph, Congresus Numerantium 60 (1987), 181-188.

[Oe3] O.R. Oellermann, On the l-connectivity of a graph, Graphs and Combinatorics 3 (1987), 285-291.

[Oe4] O.R. Oellermann, Explorations into graph connectivity, The South African Mathematical Society, 20 (1988), 117-151

[Per] B. Peroche, On several sorts of connectivity, Discrete Mathematics 46 (1983), 267-277.

[PV] B. Peroche and C. Virlouvet, Minimally 4-edge\#-connected graphs, Discrete Mathematics 125 (1994), 289-299.

[Ple] J. Plesnik, Critical graphs of given diameter, Acta Fac. Rerum Natur. Univ. Comenian Math., 30 (1975), 71-93.

[Plu] ?M.D. Plummer, On minimal blocks, Trans. Amer. Math. Soc., 134 (1968), 85-94.

[Sam] E. Sampathkumar, Connectivity of a graph - a generalization, J. combin, Information \& System Sciences, 9 (1984), 71-78.

[Sin] R. Singleton, On minimal graphs of maximum even girth, J. Combinatorial Theory, 1 (1966), 306-332.

[Wa1] M.E. Watkins, Connectivity of transitive graphs, J. Combinatorial Theory, 8 (1970), 23-29. 
[Wa2] M.E. Watkins, Some classes of hypoconnected vertex-transitive graphs, Recent Progress in Combinatorics. Academic, New York (1969), 323328.

[Wh1] H. Whitney, Congruent graphs and the connectivity of graphs, Amer. J. Math, 54 (1932), 150-168.

[Wh2] H. Whitney, Non-separable and planar graphs, Trans. Amer. Math. Soc., 34 (1932), 339-362.

[Wil1] R.S. Wilkov, Analysis and design of reliable computer networks, IEEE Trans. Comm., COM-20 (1972), 660-678.

[Wil2] R.S. Wilkov, On maximally connected graphs of minimal diameter, Proc, 1970 IEEE Intermat. Symp. Circuit Theory (Dec. 1970), 25-26.

[Wil3] R.S. Wilkov, Construction of optimal graph theoretic codes, Doctoral dissertation, Columbia Univ., New York, 1969.

[ZX] F. Zhang and X. Guo, Reducible chains in several types of 2-connected graphs*, Discrete Mathematics 105 (1992), 285-291.

[Zha] Y. Zhao, Edge-reconstruction of minimally 3-connected planar graphs, Aequationes Mathematics 47 (1994), 1-10. 


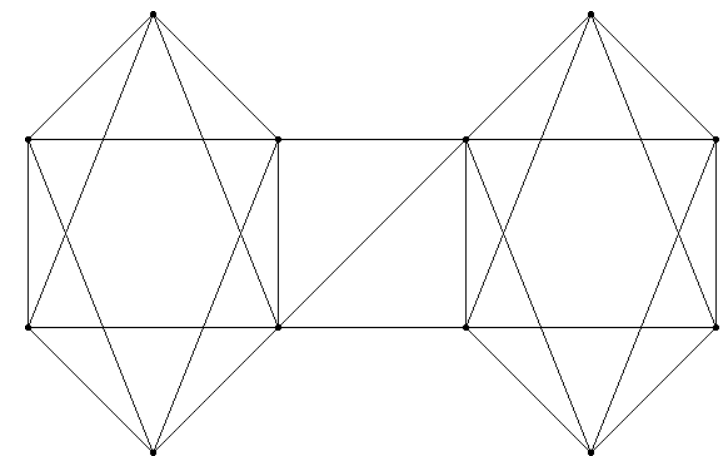

Figure 3.1. Example of a graph in which $\kappa \neq \lambda$.

$$
\kappa=2 \text { and } \lambda=3 \text {. }
$$

BACK 


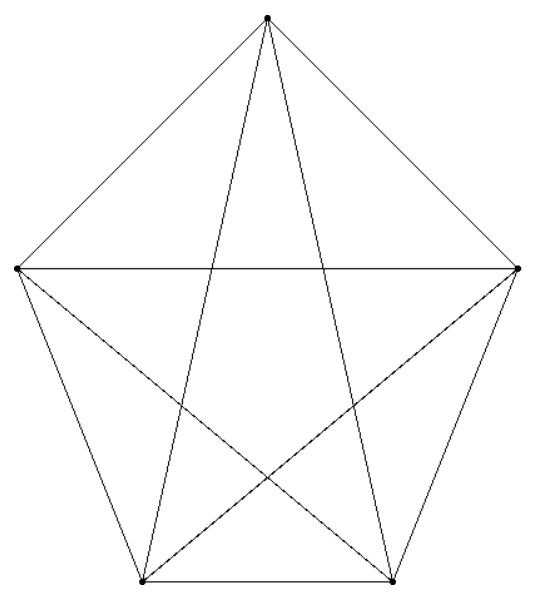

Figure 3.2. Circulant graph $\mathrm{C}_{5}[1,2]$.

Note that for this graph $\mathrm{a}_{1}=1, \mathrm{a}_{2}=2$ and $\mathrm{k}=2$.

Degree $=2 \mathrm{k}=4$ and $\lambda=4$.

BACK 


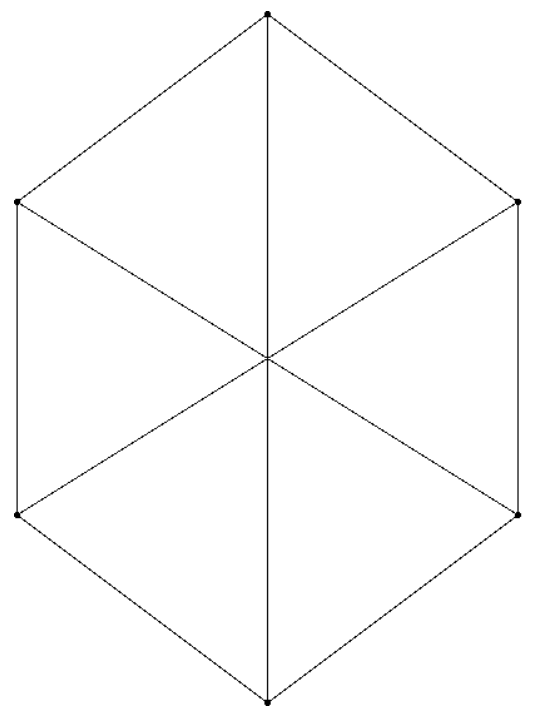

Figure 3.3. Circulant graph $\mathrm{C}_{6}[1,3]$.

Note that for this graph $\mathrm{a}_{1}=1, \mathrm{a}_{2}=3$ and $\mathrm{k}=2$.

Degree $=2 \mathrm{k}-1=3$ and $\lambda=3$.

BACK 


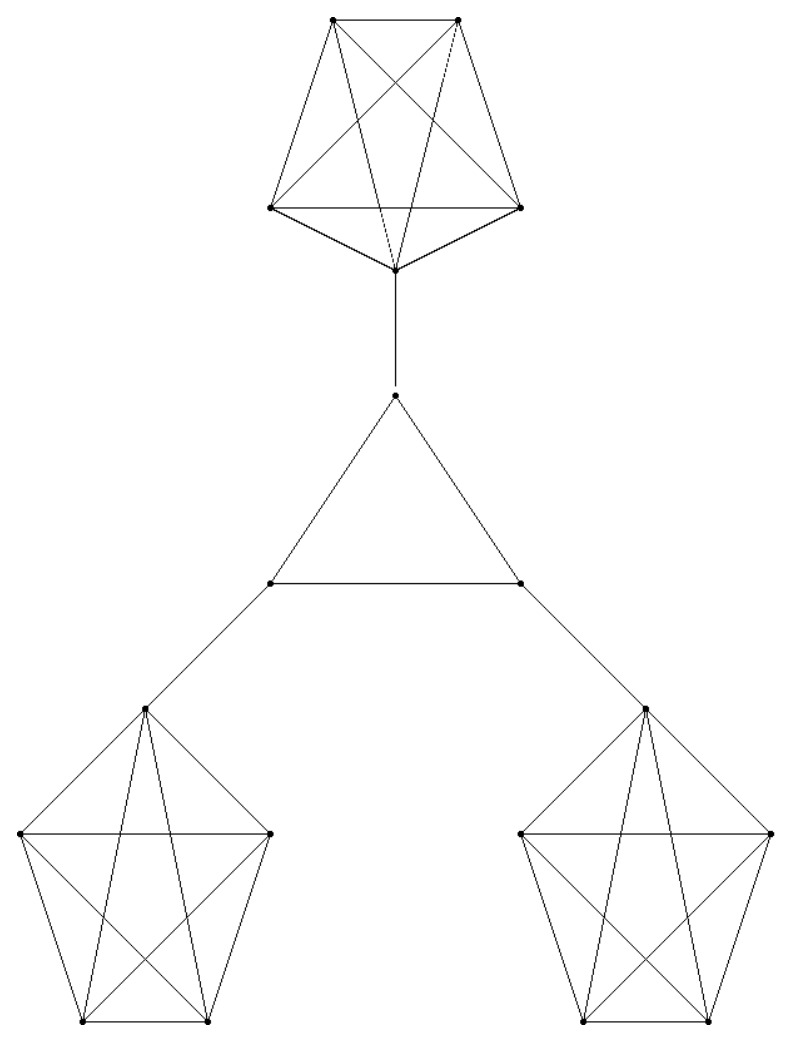

Figure 3.4. Generalized edge-connectivities. $\lambda_{2}=1, \lambda_{3}=2$ and $\lambda_{4}=3$.

BACK 


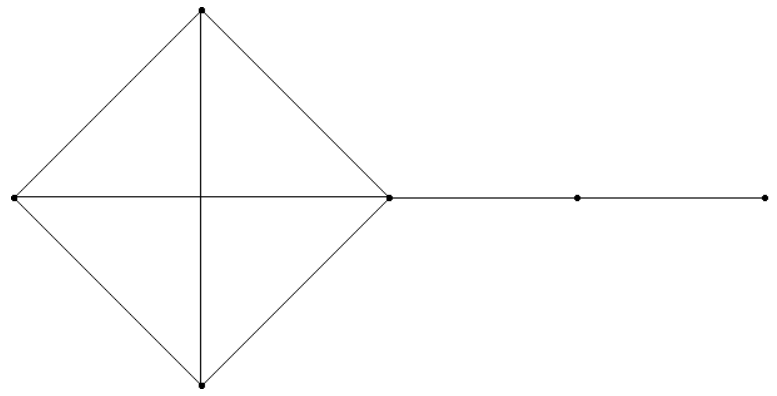

Figure 3.5. Example of a graph in which $\kappa_{1}>\lambda_{1}$ for $1>2$. $\lambda_{3}=2$ and $\kappa_{3}=4$.

\section{BACK}




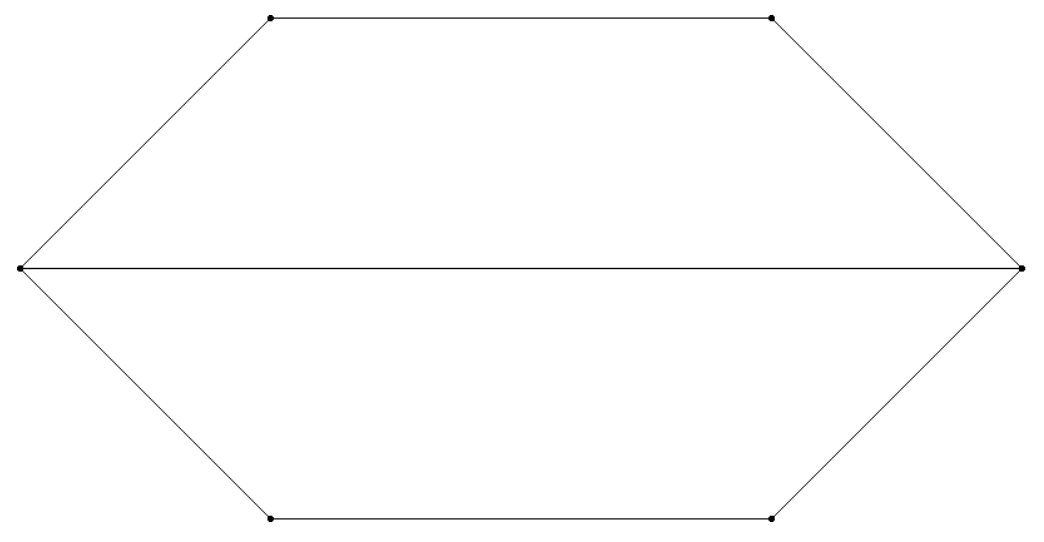

Figure 3.6. A critically 2-edge-connected graph but it is not minimally 2-edge-connected.

BACK 


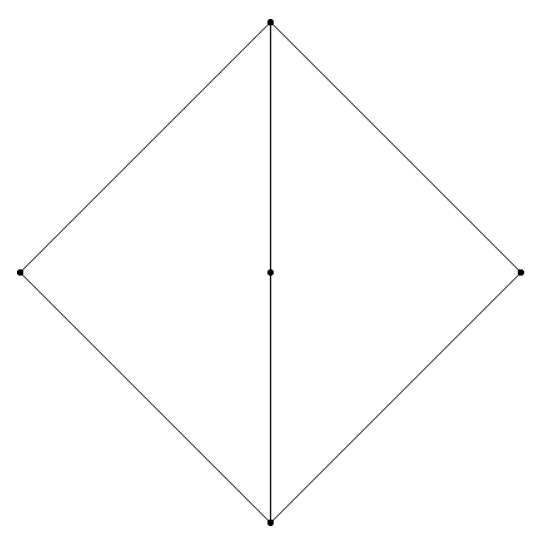

Figure 3.7. A minimally 2-edge-connected graph but it is not critically 2-edge-connected.

BACK 


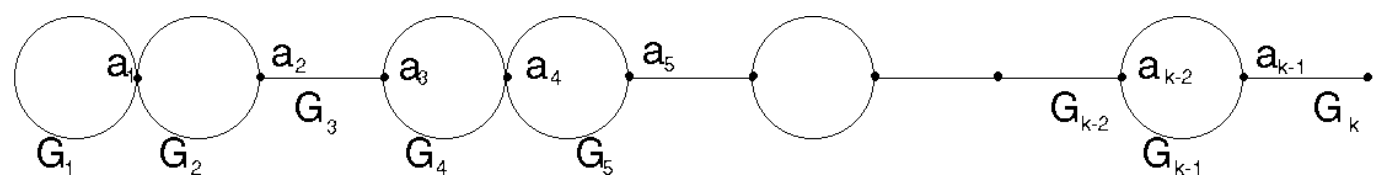

Figure 3.8. E-chain graph.

BACK 


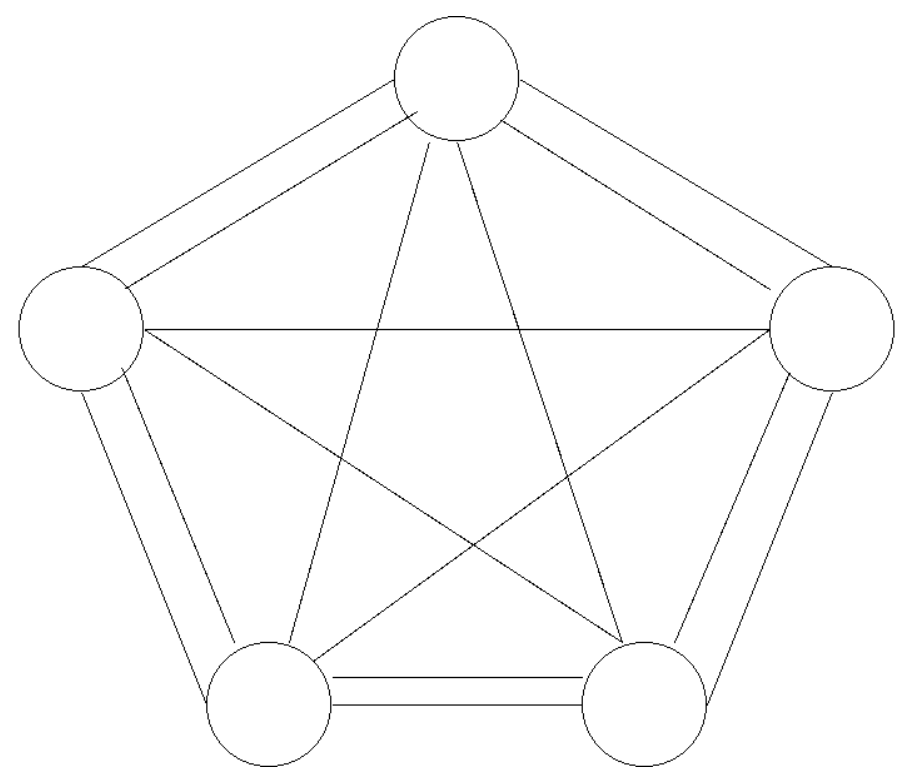

Figure 4.1. Circulant component graph $\mathrm{CC}_{5}[1(2), 2(1)]$.

BACK 


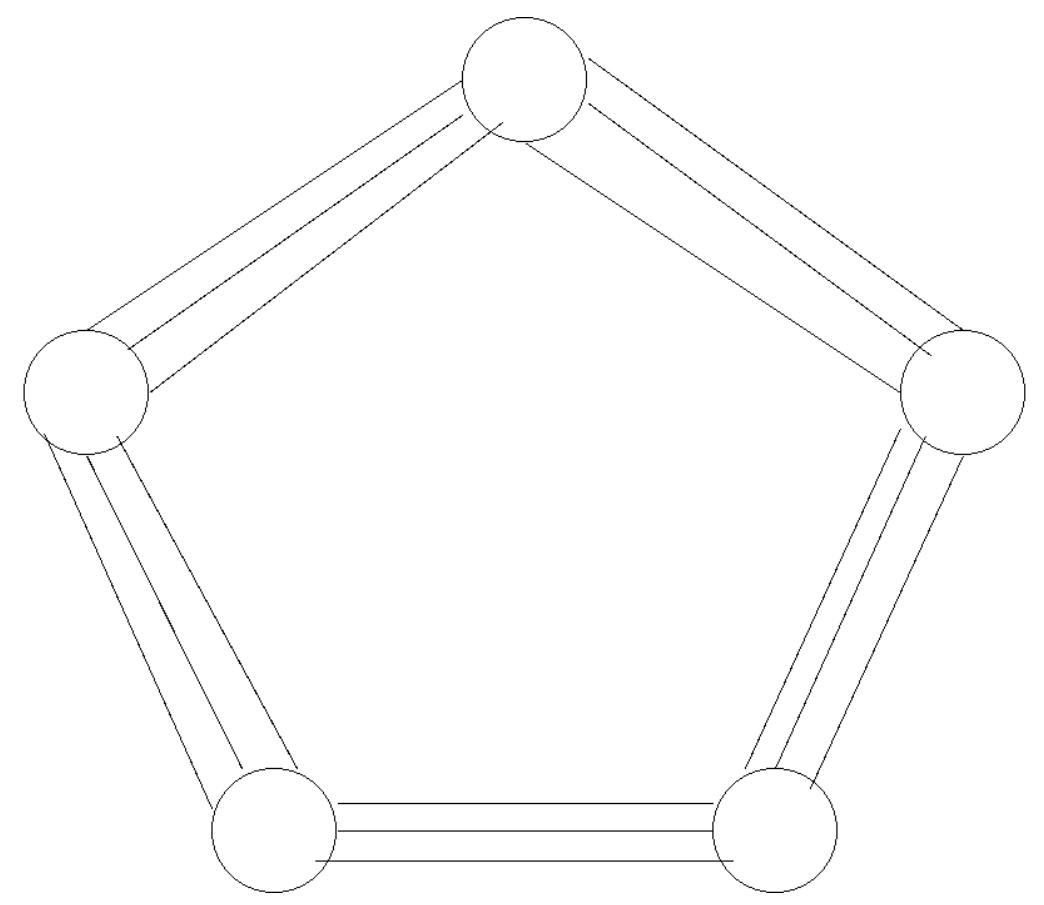

Figure 4.2. Circulant component graph $\mathrm{CC}_{5}[1(3)]$.

\section{BACK}




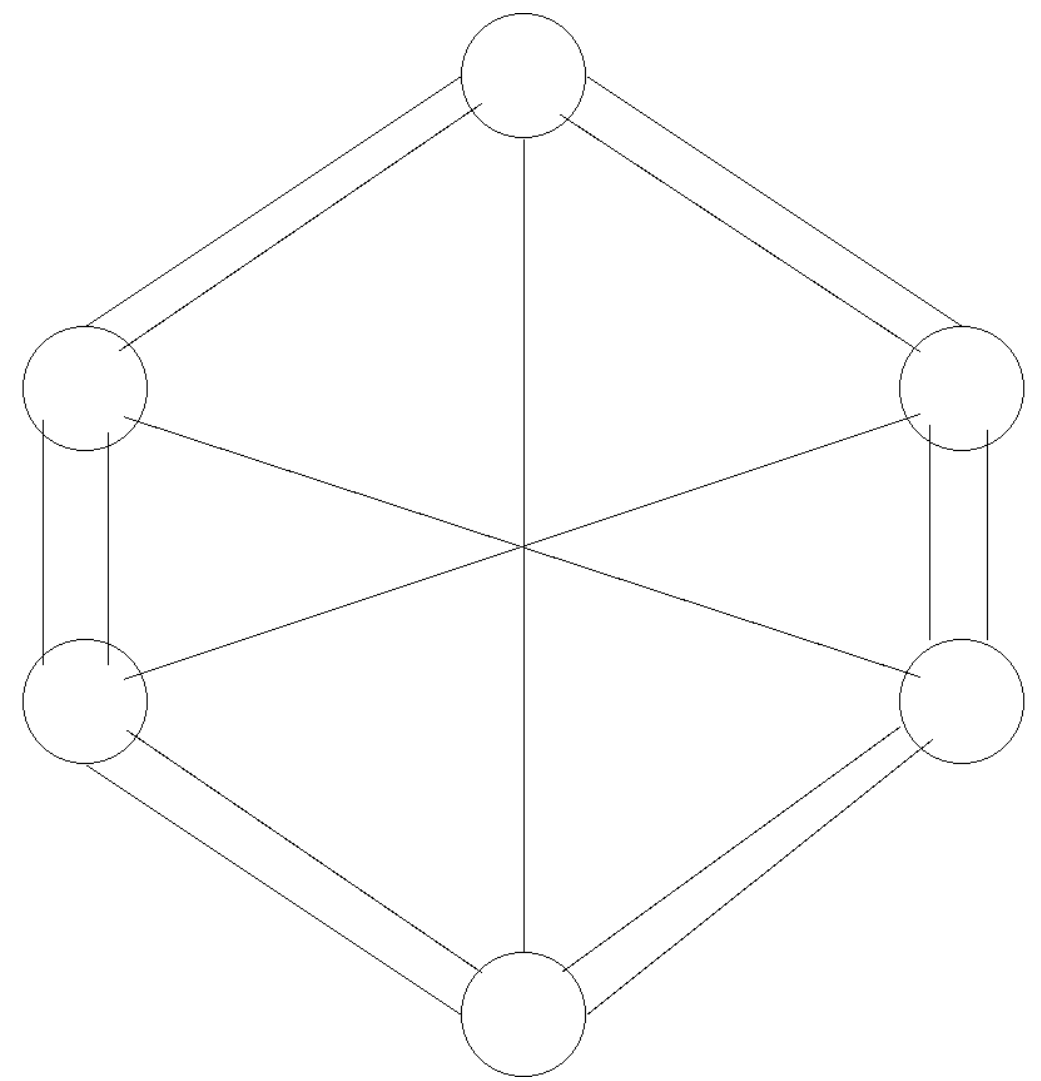

Figure 4.3. Circulant component graph CC6[1(2), 3(1)].

BACK 


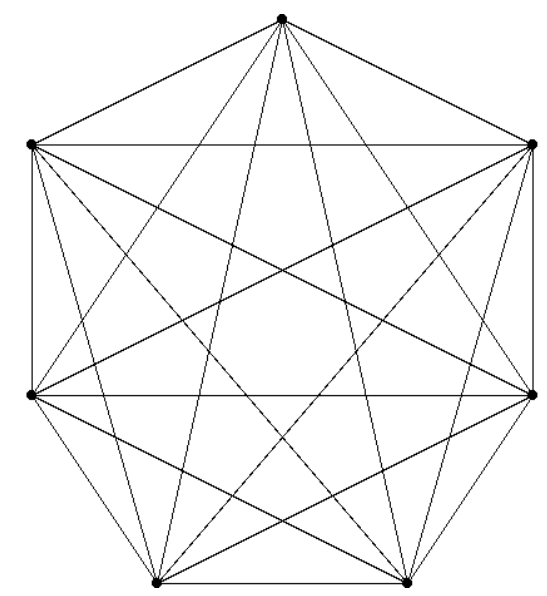

Figure 4.4(a). The circulant graph $C_{7}[1,2,3] \cong K_{7}$.

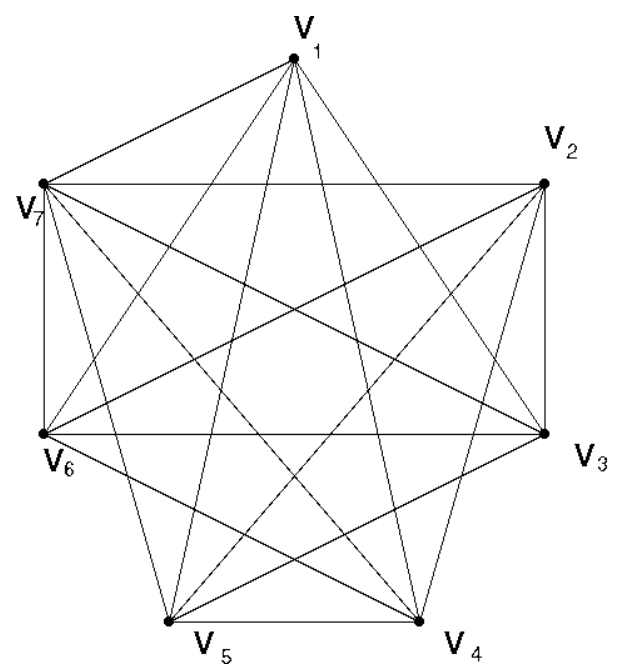

Figure 4.4(b). The graph $C_{7}[1,2,3]-\left\{v_{1} v_{2}, v_{3} v_{4}, v_{5} v_{6}\right\}$.

BACK 


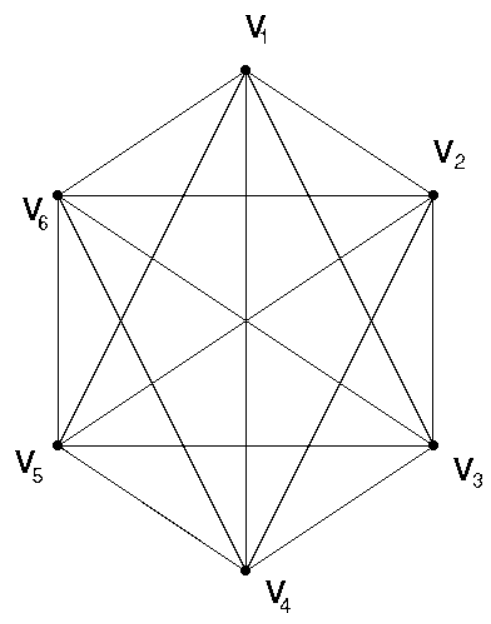

Figure 4.5(a). The circulant graph $\mathrm{C}_{6}[1,2,3] \cong \mathrm{K}_{6}$.

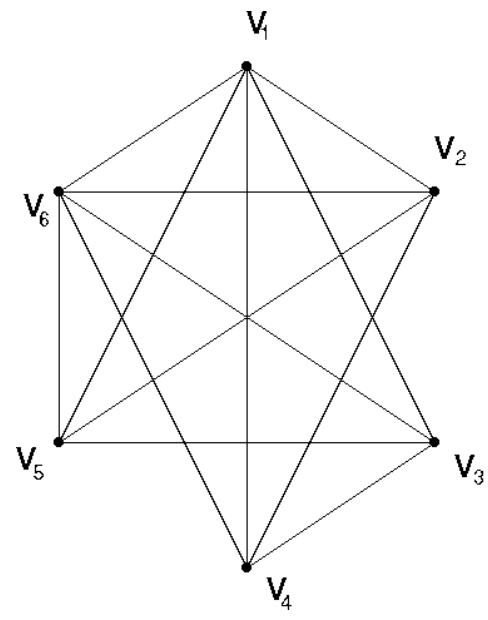

Figure 4.5(b). The graph $\mathrm{C}_{6}[1,2,3]-\left\{\mathrm{v}_{2} \mathrm{v}_{3}, \mathrm{v}_{4} \mathrm{v}_{5}\right\}$.

BACK 


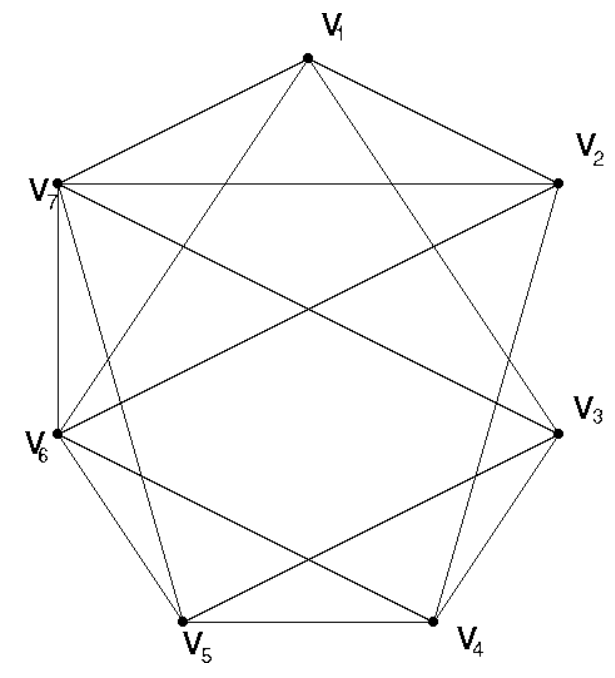

Figure 4.6. The graph $C_{7}[1,2]+\left\{v_{2} v_{6}, v_{3} v_{7}\right\}-\left\{v_{2} v_{3}\right\}$.

BACK 


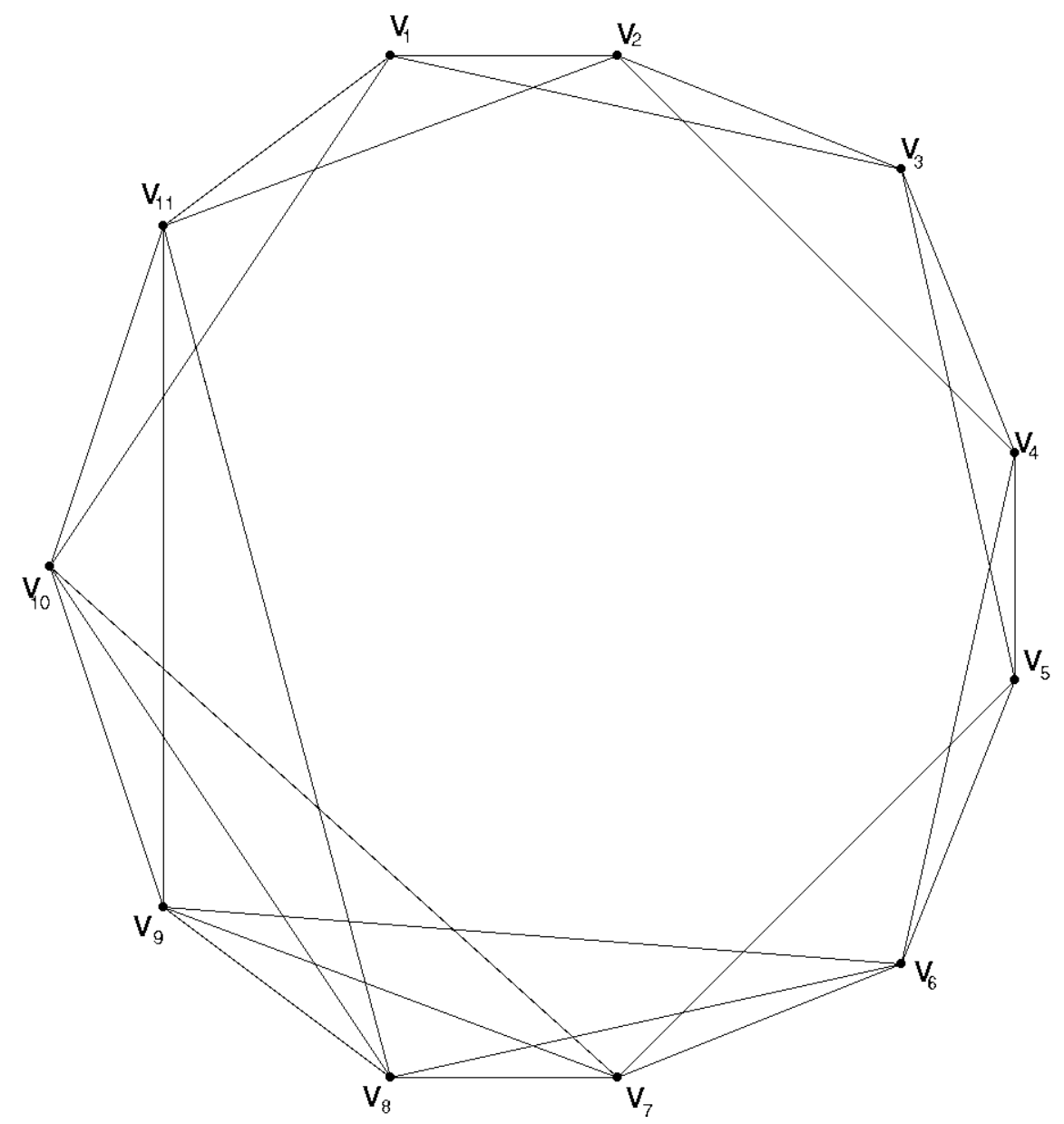

Figure 4.7. The graph $\mathrm{C}_{11}[1,2]+\left\{\mathrm{v}_{6} \mathrm{~V}_{9}, \mathrm{v}_{7} \mathrm{~V}_{10}, \mathrm{~V}_{8} \mathrm{~V}_{11}\right\}$.

BACK 